\title{
Dufour and Soret Effect on Viscous Fluid Flow between Squeezing Plates under the Influence of Variable Magnetic Field
}

\author{
Muhammad Kamran Alam ${ }^{1, *}$, Khadija Bibi ${ }^{1}$, Aamir Khan ${ }^{1}$ and Samad Noeiaghdam ${ }^{2,3}$ (1) \\ 1 Department of Pure and Applied Mathematics, The University of Haripur, Haripur 22620, Pakistan; \\ p109954@nu.edu.pk (K.B.); aamir.khan@uoh.edu.pk (A.K.) \\ 2 Industrial Mathematics Laboratory, Baikal School of BRICS, Irkutsk National Research Technical University, \\ 664074 Irkutsk, Russia; snoei@istu.edu or noiagdams@susu.ru \\ 3 Department of Applied Mathematics and Programming, South Ural State University, Lenin Prospect 76, \\ 454080 Chelyabinsk, Russia \\ * Correspondence: mkalam@uoh.edu.pk
}

Citation: Alam, M.K.; Bibi, K.; Khan, A.; Noeiaghdam, S. Dufour and Soret Effect on Viscous Fluid Flow between Squeezing Plates under the Influence of Variable Magnetic Field. Mathematics 2021, 9, 2404. https://doi.org/10.3390/math9192404

Academic Editors: Vladimir Iosifovich Semenov and Almudena del Pilar Marquez Lozano

Received: 14 August 2021

Accepted: 19 September 2021

Published: 27 September 2021

Publisher's Note: MDPI stays neutral with regard to jurisdictional claims in published maps and institutional affiliations.

Copyright: (c) 2021 by the authors. Licensee MDPI, Basel, Switzerland. This article is an open access article distributed under the terms and conditions of the Creative Commons Attribution (CC BY) license (https:// creativecommons.org/licenses/by/ $4.0 /)$.

\begin{abstract}
The aim of this article is to investigate the effect of mass and heat transfer on unsteady squeeze flow of viscous fluid under the influence of variable magnetic field. The flow is observed in a rotating channel. The unsteady equations of mass and momentum conservation are coupled with the variable magnetic field and energy equations. By using some appropriate similarity transformations, the partial differential equations obtained are then converted into a system of ordinary differential equations and are solved by Homotopy Analysis Method (HAM). The influence of the natural parameters are investigated for the velocity field components, magnetic field components, heat and mass transfer. A direct effect of the squeeze Reynold number is observed on both concentration and temperature. Moreover, increasing the magnetic Reynold number shows an increase in the fluid temperature, but in the case of concentration, an inverse relation is observed. Furthermore, a decreasing effect of the Dufour number is observed on both concentration and temperature distribution. Besides, in case of the Soret number, a direct effect is observed on concentration, but an inverse effect can be seen on temperature distribution. Different effects are shown through graphs in this study and an error analysis is also presented through tables and graphs.
\end{abstract}

Keywords: unsteady squeeze flow; viscous fluid; heat transfer; mass transfer; Dufour effect; Soret effect; HAM

\section{Introduction}

The flow of a fluid squeezed between two parallel plates approaching one another is called a squeeze flow. The unsteady squeezing flow between two rotating plates is regarded as one of the most important study areas due to its extensive applications in science and technology. Among these are hydrodynamic lubrication, polymer technology, biomechanics and aerodynamic heating. The interaction of conducting fluids with electromagnetic fields is widely known as Magento-Hydro Dynamics (MHD). The use of an MHD fluid as a lubricant in industrial applications is appealing because it prevents the unanticipated variation of lubricant viscosity with temperature under such high working conditions, and thus, it has gained the interest of many researchers, as the unsteady squeezing flow between parallel plates was considered for viscous MHD fluid by Siddiqui et al. [1]. Further, Erik Sweet [2] investigated the analytical solution for a viscous fluid flow between moving parallel plates in an unstable MHD flow. They used the homotopy analysis method to find the solution, which indicated that the magnetic field's strength has a significant impact on the flow. Later on, Murty et al. [3] observed the electrically conducting fluid in a two-phase MHD convective flow under the action of a constant transverse magnetic field through an inclined channel in a rotating system. Onyango et al. [4] discussed an unsteady MHD flow of 
viscous fluid between two parallel plates under a constant pressure gradient. Khan et al. [5] observed the flow of a viscous fluid between compressing parallel plates under the influence of a varying magnetic field. They investigated the entropy generation due to magnetic fields, fluid friction and heat transfer in a two-dimensional flow problem. Muhammad et al. [6] discussed the squeezing MHD flow between two parallel plates using Jeffrey fluid. MHD fluid flow between two parallel plates was investigated by Verma et al. [7]. Later on, Hayat et al. [8] analytically treated the squeeze flow of MHD nano fluids between two parallel plates. Moreover, T. Linga Raju [9] discussed the MHD two fluid flow of ionized gases and investigated the effect of hall current on temperature distribution. The effect of magnetohydrodynamics on a fluid film was then observed by E A Hamza [10]; he studied the squeezed flow between two surfaces while rotation was added to the surfaces. Unsteady Couette flow was then studied by Das et al. [11], where the flow was unsteady and the MHD effect was added. The flow was observed in a rotating system.

A viscous fluid flow between rotating parallel plates with varying but constant angular velocities was investigated by Parter et al. [12]. In addition, [13] also made some observations about the flow of viscous fluid between two parallel rotating plates. Further on, [14] K.R. Rajagopal also studied second ordered fluid flowing in a rotating system, and later on, the MHD double diffusive flow of nanofluids was studied by Tripathi et al. [15], where the flow was observed in a rotating channel with viscous dissipation and hall effect.

The MHD flow of viscous fluids in a rotating frame was also studied in cylindrical coordinates as was discussed by Hughes et al. [16]. They examined the lubrication flow of such viscous fluids between rotating parallel disks. In addition, Elshekh et al. [17] observed the flow of fluid film squeezed between rotating parallel disks where an external magnetic field was applied. The influence of a changing magnetic field on the unsteady squeezing flow of viscous fluids between rotating discs was also examined by Shah et al. [18]. The squeezing unsteady flow of MHD fluid between two disks was also discussed by Ganji et al. [19], who observed the flow with suction or injection involved. Between squeezing discs moving at various velocities, the effects of MFD viscosity and magnetic-field-based (MFD) thermosolutal convection of the fluid dynamics are examined by Khan et al. [20].

The unsteady squeeze flow of viscous fluids has been studied in three-dimensional rotating systems by several researchers. Recently, Munawar et al. [21] studied the squeeze flow of viscous fluids in a three-dimensional rotating system. The flow was considered between parallel plates with the lower stretching plate kept porous. Further on, Alzahrani et al. [22] numerically treated the squeezed flow of viscous fluid between rotating parallel plates in a three-dimensional system and examined the effect of Dufour and Soret numbers. Similar work has been done on third grade nanofluids in a three-dimensional rotating system where the thermophoresis effect and Brownian motion was observed by Shah et al. [23]. In addition, the thin film flow of Darcy Forchheimer hydromagnetic nanofuid between rotating parllel disks in a three-dimensional system was discussed by Riasat et al. [24]. They examined the importance of the magnetic Reynold number in such a system. Moreover, Fiza et al [25] examined the flow of Jeffrey fluid in a three-dimensional rotating system.

Srinivas et al. [26] studied the impact of heat transfer on the flow of MHD fluid through a pipe whose walls are expanding or contracting. The simultaneous effect of induced magnetic forces and buoyancy forces on heat transfer was investigated by Sparrow et al. [27]. Furthermore, Khaled et al. [28] discussed the flow of fluid and its heat transfer over a horizontal surface, placed in a free stream, externally squeezed. The effect of Radiation on MHD fluid and its mass transfer was studied by Hayat et al. [29], who observed the flow on a stretching sheet which was kept porous. Mahmood et al. [30] analyzed the flow of viscous fluid over a permeable sensor surface with the squeeze effect and also observed the heat transfer effect for such a flow phenomenon. In addition, the Hiemenz flow over a stretching surface was examined by Tsai et al. [31], where the medium was porous. The heat and mass transfer was studied theoretically for Dufour's and Soret effect. The Heat and Mass transfer of a viscous fluid between squeezing plates was further 
observed by Mustafa et al. [32]. Furthermore, Yasmin et al. [33] studied non-Newtonian micropolar fluids in a magnetohydrodynamics flow on a curved sheet with stretching effect and discussed the mass and heat transfer for such fluids. Heat and mass transfer in a three-dimensional flow of nanofluids in a rotating system was examined by Sheikholeslami et al. [34]. Noeiaghdam et al. studied the numerical analysis of a natural convection-driven flow of a non-Newtonian power-law fluid in a trapezoidal enclosure with a U-Shaped constructal [35-39].

The above existing literature shows that no study has been conducted so far on the three-dimensional squeeze flow of viscous fluids between two parallel rotating plates under the influence of the variable magnetic field. Additionally, the effect of variable magnetic field on mass transfer in Cartesian coordinates is new work in this area. Hence, the suggested work is the best approach toward such problems and is a way of motivation for researchers bringing a new idea of studying the flow between unsteady rotating parallel plates.

\section{Modeling and Formulation of the Physical Problem}

We consider an incompressible viscous fluid flow between two squeezing plates, separated by a distance $D(t)=l(1-\beta t)^{1 / 2}$, where $l$ is the spacing between plates at time $t=0$ and $\beta$ is the expansion and contraction parameter whose values lies between $(0,1)$ for squeezing phenomenon, as shown in Figure 1. With an angular velocity of $\Omega_{u}$, the upper plate rotates whereas the lower plate is moving with angular velocity $\Omega_{l}$. The effect of variable magnetic field $M$ is added externally which produces the induced magnetic field $B$ with the following components: $B_{x}, B_{y}$ and $B_{z}$.

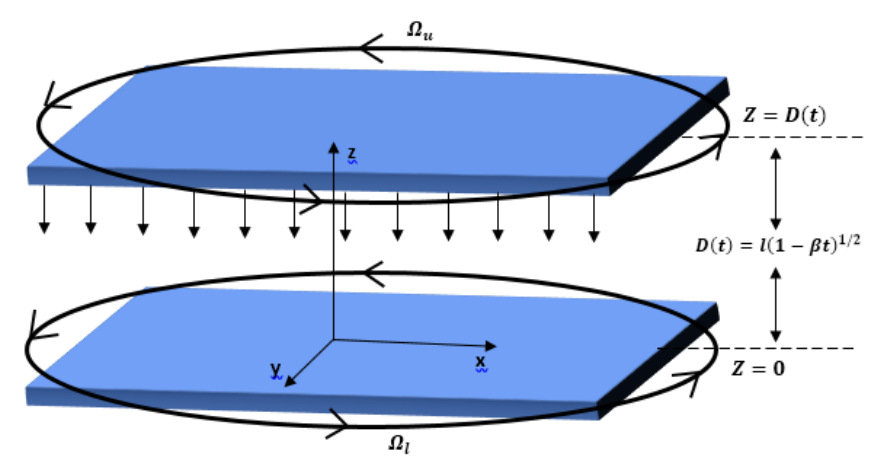

Figure 1. Geometry of the flow problem.

The system of coordinates selected is Cartesian coordinates. The origin is fixed at the center of the lower plate, in which the $\mathrm{x}$-axis is taken along the horizontal axis and the $z$-axis is perpendicular to the two plates (along the vertical axis). The rotation of plates is along the y-axis. The flow between the plates occurs due to the rotatory motion of plates and the motion of upper plate towards the lower plate, i.e., the squeezing effect. The effect of gravity on fluid is negligible. Now, we will observe the velocity profile of the given fluid, the effect of magnetic field on the velocity of fluid, and the heat and mass transfer for these viscous fluids in a three-dimensional system. Thus, the vector form of continuity, momentum, magnetic field, energy and concentration equations are given by:

$$
\frac{\partial u}{\partial x}+\frac{\partial v}{\partial y}+\frac{\partial w}{\partial z}=0
$$


Navier-Stokes equation $\mathrm{x}$-component:

$$
\begin{gathered}
\rho\left[\frac{\partial u}{\partial t}+u \frac{\partial u}{\partial x}+v \frac{\partial u}{\partial y}+w \frac{\partial u}{\partial z}\right]=-\frac{\partial P}{\partial x}+\mu\left[\frac{\partial^{2} u}{\partial x^{2}}+\frac{\partial^{2} u}{\partial y^{2}}+\frac{\partial^{2} u}{\partial z^{2}}\right]+ \\
\frac{1}{\mu_{2}}\left[B_{z} \frac{\partial B_{x}}{\partial z}-B_{z} \frac{\partial B_{z}}{\partial x}-B_{y} \frac{\partial B_{y}}{\partial x}+B_{y} \frac{\partial B_{x}}{\partial y}\right]
\end{gathered}
$$

Navier-Stokes equation y-component:

$$
\begin{gathered}
\rho\left[\frac{\partial v}{\partial t}+u \frac{\partial v}{\partial x}+v \frac{\partial v}{\partial y}+w \frac{\partial v}{\partial z}\right]=-\frac{\partial p}{\partial y}+\mu\left[\frac{\partial^{2} v}{\partial x^{2}}+\frac{\partial^{2} v}{\partial y^{2}}+\frac{\partial^{2} v}{\partial z^{2}}\right]+ \\
\frac{1}{\mu_{2}}\left[B_{x} \frac{\partial B_{y}}{\partial x}-B_{x} \frac{\partial B_{x}}{\partial y}-B_{z} \frac{\partial B_{z}}{\partial y}+B_{z} \frac{\partial B_{y}}{\partial z}\right]
\end{gathered}
$$

Navier-Stokes equation z-component:

$$
\begin{gathered}
\rho\left[\frac{\partial w}{\partial t}+u \frac{\partial w}{\partial x}+v \frac{\partial w}{\partial y}+w \frac{\partial w}{\partial z}\right]_{=}=-\frac{\partial p}{\partial z}+\mu\left[\frac{\partial^{2} w}{\partial x^{2}}+\frac{\partial^{2} w}{\partial y^{2}}+\frac{\partial^{2} w}{\partial z^{2}}\right]+ \\
\frac{1}{\mu_{2}}\left[B_{y} \frac{\partial B_{z}}{\partial y}-B_{y} \frac{\partial B_{y}}{\partial z}-B_{x} \frac{\partial B_{x}}{\partial z}+B_{x} \frac{\partial B_{z}}{\partial x}\right]
\end{gathered}
$$

Magnetic field equation $x$-component:

$$
\begin{gathered}
\frac{\partial B_{x}}{\partial t}=\left[u \frac{\partial B_{y}}{\partial y}+B_{y} \frac{\partial u}{\partial y}-v \frac{\partial B_{x}}{\partial y}-B_{x} \frac{\partial v}{\partial y}-w \frac{\partial B_{x}}{\partial z}-B_{x} \frac{\partial w}{\partial z}+u \frac{\partial B_{z}}{\partial z}+B_{z} \frac{\partial u}{\partial z}\right]+ \\
\frac{1}{\delta \mu_{2}}\left[\frac{\partial^{2} B_{x}}{\partial x^{2}}+\frac{\partial^{2} B_{x}}{\partial y^{2}}+\frac{\partial^{2} B_{x}}{\partial z^{2}}\right]
\end{gathered}
$$

Magnetic field equation y-component:

$$
\begin{gathered}
\frac{\partial B_{y}}{\partial t}=\left[v \frac{\partial B_{z}}{\partial z}+B_{z} \frac{\partial v}{\partial z}-w \frac{\partial B_{y}}{\partial z}-B_{y} \frac{\partial w}{\partial z}-u \frac{\partial B_{y}}{\partial x}-B_{y} \frac{\partial u}{\partial x}+v \frac{\partial B_{x}}{\partial x}+B_{x} \frac{\partial v}{\partial x}\right]+ \\
\frac{1}{\delta \mu_{2}}\left[\frac{\partial^{2} B_{y}}{\partial x^{2}}+\frac{\partial^{2} B_{y}}{\partial y^{2}}+\frac{\partial^{2} B_{y}}{\partial z^{2}}\right]
\end{gathered}
$$

Magnetic field equation z-component:

$$
\begin{gathered}
\frac{\partial B_{z}}{\partial t}=\left[w \frac{\partial B_{x}}{\partial x}+B_{x} \frac{\partial w}{\partial x}-u \frac{\partial B_{z}}{\partial x}-B_{z} \frac{\partial u}{\partial x}-v \frac{\partial B_{z}}{\partial y}-B_{z} \frac{\partial v}{\partial y}+w \frac{\partial B_{y}}{\partial y}+B_{y} \frac{\partial w}{\partial y}\right]+ \\
\frac{1}{\delta \mu_{2}}\left[\frac{\partial^{2} B_{z}}{\partial x^{2}}+\frac{\partial^{2} B_{z}}{\partial y^{2}}+\frac{\partial^{2} B_{z}}{\partial z^{2}}\right]
\end{gathered}
$$

Heat transfer equation:

$$
\begin{gathered}
\rho\left[\frac{\partial T}{\partial t}+u \frac{\partial T}{\partial x}+v \frac{\partial T}{\partial y}+w \frac{\partial T}{\partial z}\right]=\frac{\kappa}{C_{p}}\left[\frac{\partial^{2} T}{\partial x^{2}}+\frac{\partial^{2} T}{\partial y^{2}}+\frac{\partial^{2} T}{\partial z^{2}}\right]+ \\
\frac{1}{C_{p}} \nabla q_{r}+\frac{D \kappa_{t}}{C_{s} C_{p}}\left[\frac{\partial^{2} C}{\partial x^{2}}+\frac{\partial^{2} C}{\partial y^{2}}+\frac{\partial^{2} C}{\partial z^{2}}\right]
\end{gathered}
$$

and similarly, the equation for mass transfer is given by

$$
\begin{gathered}
\rho\left[\frac{\partial C}{\partial t}+u \frac{\partial C}{\partial x}+v \frac{\partial C}{\partial y}+w \frac{\partial C}{\partial z}\right]=D\left[\frac{\partial^{2} C}{\partial x^{2}}+\frac{\partial^{2} C}{\partial y^{2}}+\frac{\partial^{2} C}{\partial z^{2}}\right]+ \\
\frac{D \kappa_{t}}{C_{s} T_{m}}\left[\frac{\partial^{2} T}{\partial x^{2}}+\frac{\partial^{2} T}{\partial y^{2}}+\frac{\partial^{2} T}{\partial z^{2}}\right] .
\end{gathered}
$$


where $\rho$ is density of fluid, $\mu_{2}$ is permittivity of free space, $\mu$ is viscosity, $P$ is pressure, $V$ is velocity of fluid, $B$ is magnetic field, $T$ is temperature, $C$ is concentration, $\kappa_{t}$ is thermal diffusion ratio, $D$ is diffusion coefficient, $C_{p}$ is specific heat at constant pressure, $\kappa$ is conductivity and $\nabla q_{r}$ is the radiative heat flux, given as,

$$
\nabla q_{r}=\frac{16 \sigma^{s} T_{u}^{3}}{3 \kappa^{a}} \frac{\partial^{2} T}{\partial z^{2}}
$$

Here, $\kappa^{a}$ and $\sigma^{s}$ are the mean absorption coefficient and Stefan-Boltzmann constant, respectively. The boundary conditions for the above fluid flow are given as follows, at lower plate where $z=0, u=0, v=\frac{\Omega_{l} x}{1-\beta t}, w=0, B_{x}=B_{y}=B_{z}=0, T=T_{l}, C=C_{l}$.

Moreover, observe the conditions at upper plate where $z=D(t), u=0, v=\frac{\Omega_{u} x}{1-\beta t^{\prime}}$, $w=\frac{d D(t)}{d t}, B_{x}=0 B_{y}=\frac{x N_{0}}{1-\beta t}, B_{z}=\frac{-\beta M_{0}}{(1-\beta t)^{1 / 2}}, T=T_{u}, C=C_{u}$. where $D(t)=$ $l(-\beta t)^{1 / 2}$. Now, using the following transformation to convert the above partial differential equations to ordinary differential equations (ODEs), $u=\frac{\beta x}{(1-\beta t)} f^{\prime}(\eta), v=\frac{\Omega_{l} x}{(1-\beta t)} g(\eta)$, $w=\frac{-\beta l}{(1-\beta t)^{1 / 2}} f(\eta), B_{x}=\frac{\beta x M_{0}}{l(1-\beta t)} m^{\prime}(\eta), B_{y}=\frac{x N_{0}}{(1-\beta t)} n(\eta), B_{z}=\frac{-\beta M_{0}}{(1-\beta t)^{1 / 2}} m(\eta)$, $\theta=\frac{T-T_{u}}{T_{l}-T_{u}}, \phi=\frac{C-C_{u}}{C_{l}-C_{u}}, \eta=\frac{z}{l(1-\beta t)^{1 / 2}}$.

After non-dimensionlizing the above equations and setting boundary conditions, they will be converted to the following ODEs,

$$
\begin{gathered}
f^{\prime \prime \prime \prime}=S_{z}\left[3 f^{\prime \prime}+(\eta-2 f) f^{\prime \prime \prime}-2 f^{\prime} f^{\prime \prime}\right]+ \\
2 S_{z} M_{x}^{2}\left[2 R_{m}\left(m m^{\prime}+\eta m m^{\prime \prime}-f m m^{\prime \prime}+m^{2} f^{\prime \prime}\right)-m^{\prime} m^{\prime \prime}\right], \\
g^{\prime \prime}=S_{z}\left[2 g+\eta g^{\prime}+2 f^{\prime} g-2 g^{\prime} f\right]-2 S_{z} M_{x} M_{y}\left[m^{\prime} n-n^{\prime} m\right], \\
m^{\prime \prime}=R_{m}\left[m+\eta m^{\prime}-2 m^{\prime} f+2 f^{\prime} m\right], \\
n^{\prime \prime}=R_{m}\left[2 n+\eta n^{\prime}-2 n^{\prime} f+2 f^{\prime} n-2\left(\frac{M_{x}}{M_{y}}\right)\left(g^{\prime} m-m^{\prime} g\right)\right], \\
\theta^{\prime \prime}\left(3+4 R_{d}\right)+3 D_{u} P_{r n} \phi^{\prime \prime}+3 S_{z} P_{r n}\left(f \theta^{\prime}-\eta \theta^{\prime}\right)=0, \\
\phi^{\prime \prime}+S_{r t} S_{c h} \theta^{\prime \prime}+S_{z} S_{c h}\left(2 f \phi^{\prime}-\eta \phi^{\prime}\right)=0 .
\end{gathered}
$$

where $S_{z}=\frac{\beta l^{2}}{2 v}$ denotes the squeezing Reynold number, $M_{x}=\frac{M_{0}}{l \sqrt{\rho \mu_{2}}}$ represents the magnetic field strength along x-axis, $M_{y}=\frac{N_{0}}{\Omega_{l} \sqrt{\rho \mu_{2}}}$ is the magnetic field strength along x-axis, $R_{m}=S_{z} B_{t}$ is given by $R_{m}=\left(\frac{\beta l^{2}}{2 v}\right)\left(v \sigma \mu_{2}\right), R_{d}=\frac{4 \sigma^{s} T_{u}^{3}}{K^{a} K}$ is the radiation parameter, $D_{u}=\frac{D K_{T} K\left(C_{l}-C_{u}\right)}{C_{s} v C_{p}\left(T_{l}-T_{u}\right) v}$ is Dufour number and $P_{r n}=\frac{v C_{p}}{K}$ is Prandtl number. The boundary conditions become the following forms, $f(0)=0, f^{\prime}(0)=0, g(0)=1, m(0)=0, n(0)=0$, $\theta(0)=1, \phi(0)=1 . f(1)=\frac{1}{2}, f^{\prime}(1)=0, g(1)=\frac{\Omega_{u}}{\Omega_{l}}=S, m(1)=1, n(1)=1, \theta(1)=0$, $\phi(1)=0$. 


\section{Method of Solution}

An analytical technique is used to find the solution of the Equations (11)-(16), known as the Homotopy Analysis Method. We will express the functions $f, g, m, n, \theta$ and $\phi$ (where $f, g, m, n, \theta$ and $\phi$ are the functions of $\eta)$, and $\eta^{q^{*}}, q^{*} \geq 0$ are used as a set of base functions,

$$
\begin{aligned}
& f_{n}=\sum_{q^{*}=0}^{\infty} a_{q^{*}} \eta^{q^{*}} \\
& g_{n}=\sum_{q^{*}=0}^{\infty} b_{q^{*}} \eta^{q^{*}} \\
& m_{n}=\sum_{q^{*}=0}^{\infty} c_{q^{*}} \eta^{q^{*}} \\
& n_{n}=\sum_{q^{*}=0}^{\infty} d_{q^{*}} \eta^{q^{*}} \\
& \theta_{n}=\sum_{q^{*}=0}^{\infty} f_{q^{*}} \eta^{q^{*}} \\
& \phi_{n}=\sum_{q^{*}=0}^{\infty} g_{q^{*}} \eta^{q^{*}}
\end{aligned}
$$

where the constant co-efficients $a_{q^{*}}, b_{q^{*}}, c_{q^{*}}, d_{q^{*}}, f_{q^{*}}$ and $g_{q^{*}}$ are to be determined. Initial approximations are chosen as follows:

$$
\begin{gathered}
f_{0}=1.5 * \eta^{2}-\eta^{3} ; \\
g_{0}=(S-1) * \eta+1 ; \\
m_{0}=\eta \\
n_{0}=\eta \\
\theta_{0}=1-\eta \\
\phi_{0}=1-\eta
\end{gathered}
$$

then, the auxiliary operators are chosen,

$$
\begin{aligned}
& £_{f}=\frac{\partial^{4}}{\partial \eta^{4}}, £_{g}=\frac{\partial^{2}}{\partial \eta^{2}}, £_{\theta}=\frac{\partial^{2}}{\partial \eta^{2}}, \\
& £_{m}=\frac{\partial^{2}}{\partial \eta^{2}}, £_{n}=\frac{\partial^{2}}{\partial \eta^{2}}, £_{\phi}=\frac{\partial^{2}}{\partial \eta^{2}}
\end{aligned}
$$

with the following properties,

$$
\begin{gathered}
£_{f}\left(q_{1^{*}}^{*} \eta^{3}+q_{2^{*}}^{*} \eta^{2}+q_{3^{*}}^{*} \eta+q_{4^{*}}^{*}\right)=0 \\
£_{g}\left(q_{5^{*}}^{*} \eta+q_{6^{*}}^{*}\right)=0 \\
£_{m}\left(q_{7^{*}}^{*} \eta+q_{8^{*}}^{*}\right)=0 \\
£_{n}\left(q_{9^{*}}^{*} \eta+q_{10^{*}}^{*}\right)=0 \\
£_{\theta}\left(q_{11^{*}}^{*} \eta+q_{12^{*}}^{*}\right)=0 \\
£_{\theta}\left(q_{13^{*}}^{*} \eta+q_{14^{*}}^{*}\right)=0
\end{gathered}
$$


where $q_{1^{*}}^{*}, q_{2^{*}}^{*}, q_{3^{*}}^{*}, q_{4^{*}}^{*}, q_{5^{*}}^{*}, q_{6^{*}}^{*}, q_{7^{*}}^{*}, q_{8^{*}}^{*}, q_{9^{*}}^{*}, q_{10^{*}}^{*}, q_{11^{*}}^{*}, q_{12^{*}}^{*}, q_{13^{*}}^{*}$ and $q_{14^{*}}^{*}$ are arbitrary constants.

We can obtain the Zeroth order deformation as:

$$
\begin{gathered}
(1-s) £_{g}\left[g(\eta ; s)-g_{0}(\eta)\right]=s \hbar_{g} \aleph_{g}[f(\eta ; s), g(\eta ; s), \\
m(\eta ; s), n(\eta ; s)] \\
(1-s) £_{m}\left[m(\eta ; s)-m_{0}(\eta)\right]=s \hbar_{m} \aleph_{m}[f(\eta ; s), m(\eta ; s)] \\
(1-s) £_{n}\left[n(\eta ; s)-n_{0}(\eta)\right]=s \hbar_{n} \aleph_{n}[f(\eta ; s), g(\eta ; s), \\
m(\eta ; s), n(\eta ; s)] \\
(1-s) £_{\theta}\left[n(\eta ; s)-\theta_{0}(\eta)\right]=s \hbar_{\theta} \aleph_{\theta}[f(\eta ; s), \theta(\eta ; s), \phi(\eta ; s)] \\
(1-s) £_{\phi}\left[n(\eta ; s)-\phi_{0}(\eta)\right]=s \hbar_{\phi} \aleph_{\phi}[f(\eta ; s), \theta(\eta ; s), \phi(\eta ; s)]
\end{gathered}
$$

From Equations (14)-(19), the nonlinear operators are defined as,

$$
\begin{aligned}
& \aleph_{f}[f(\eta ; s), m(\eta ; s)]=\frac{\partial^{4} f(\eta ; s)}{\partial \eta^{4}}-S_{z}\left(3 \frac{\partial^{2} f(\eta ; s)}{\partial \eta^{2}}+(\eta-2 f)\right. \\
& \left.\frac{\partial^{3} f(\eta ; s)}{\partial \eta^{3}}-2 \frac{\partial f(\eta ; s)}{\partial \eta} \frac{\partial^{2} f(\eta ; s)}{\partial \eta^{2}}\right)-2 S_{z} M_{z}^{2}\left(M \frac{\partial^{3} M(\eta ; s)}{\partial \eta^{3}}-\right. \\
& \left.\frac{\partial M(\eta ; s)}{\partial \eta} \frac{\partial^{2} M(\eta ; s)}{\partial \eta^{2}}\right) \\
& \aleph_{g}[f(\eta ; s), g(\eta ; s), m(\eta ; s), n(\eta ; s)]=\frac{\partial^{2} g(\eta ; s)}{\partial \eta^{2}}- \\
& S_{z}\left(2 g+\eta \frac{\partial g(\eta ; s)}{\partial \eta}+2 \frac{\partial f(\eta ; s)}{\partial \eta} g-2 \frac{\partial g(\eta ; s)}{\partial \eta} f\right)- \\
& 2 S_{z} M_{z} M_{y}\left(\frac{\partial m(\eta ; s)}{\partial \eta} n-m \frac{\partial n(\eta ; s)}{\partial \eta}\right) \\
& \aleph_{m}[f(\eta ; s), m(\eta ; s)]=\frac{\partial^{2} m(\eta ; s)}{\partial \eta^{2}}-R_{m}\left(m+\eta \frac{\partial m(\eta ; s)}{\partial \eta}\right. \\
& \left.-2\left(f \frac{\partial m(\eta ; s)}{\partial \eta}-m \frac{\partial f(\eta ; s)}{\partial \eta}\right)\right) \\
& \aleph_{n}[f(\eta ; s), g(\eta ; s), m(\eta ; s), n(\eta ; s)]=\frac{\partial^{2} n(\eta ; s)}{\partial \eta^{2}}-R(2 n+ \\
& \eta \frac{\partial n(\eta ; s)}{\partial \eta}-2\left(\frac{\partial n(\eta ; s)}{\partial \eta} f-n \frac{\partial f(\eta ; s)}{\partial \eta}\right)+ \\
& \left.2 \frac{\partial M_{z}}{\partial M_{y}}\left(\frac{\partial g(\eta ; s)}{\partial \eta} m-g \frac{\partial m(\eta ; s)}{\partial \eta}\right)\right) \\
& \aleph_{\theta}[f(\eta ; s), \theta(\eta ; s), \phi(\eta ; s)]=\frac{\partial^{2} \theta(\eta ; s)}{\partial \eta^{2}}\left(3+4 R_{d}\right)+ \\
& 3 D_{u} P_{r n} \frac{\partial^{2} \phi(\eta ; s)}{\partial \eta^{2}}+3 S_{z} P_{r n}\left(2 f \frac{\partial \theta(\eta ; s)}{\partial \eta}-\eta \frac{\partial \theta(\eta ; s)}{\partial \eta}\right) \\
& \aleph_{\phi}[f(\eta ; s), \theta(\eta ; s), \phi(\eta ; s)]=\frac{\partial^{2} \phi(\eta ; s)}{\partial \eta^{2}}+S_{r t} S_{c h} \frac{\partial^{2} \theta(\eta ; s)}{\partial \eta^{2}} \\
& +S_{z} S_{c h}\left(2 f \frac{\partial \phi(\eta ; s)}{\partial \eta}-\eta \frac{\partial \phi(\eta ; s)}{\partial \eta}\right)
\end{aligned}
$$

where $s$ is a fixed parameter, nonlinear parameters are $\aleph_{f}, \aleph_{g}, \aleph_{m}$ and $\aleph_{n}$, while $\hbar_{f}, \hbar_{g}, \hbar_{m}$, $\hbar_{n}, \hbar_{\theta}$ and $\hbar_{\phi}$ are the nonzero auxiliary parameters.

For $s=0$ and $s=1$, we have 


$$
\begin{aligned}
& f(\eta, 0)=f_{0}, \quad f(\eta, 1)=f(\eta) \\
& g(\eta, 0)=g_{0}, \quad g(\eta, 1)=g(\eta) \\
& m(\eta, 0)=m_{0}, \quad m(\eta, 1)=m(\eta) \\
& n(\eta, 0)=n_{0}, \quad n(\eta, 1)=n(\eta) \\
& \theta(\eta, 0)=\theta_{0}, \quad \theta(\eta, 1)=\theta(\eta) \\
& \phi(\eta, 0)=\phi_{0}, \quad \phi(\eta, 1)=\phi(\eta)
\end{aligned}
$$

so as $s$ varies from 0 to 1 , the exact solutions $f(\eta), g(\eta), n(\eta), n(\eta), \theta(\eta)$ and $\phi(\eta)$ can be obtained from initial guesses.

For these functions, the Taylor's series are given by:

$$
\begin{gathered}
f(\eta ; s)=f_{0}+\sum_{n=1}^{\infty} s^{n} f_{n}(\eta) \\
g(\eta ; s)=g_{0}+\sum_{n=1}^{\infty} s^{n} g_{n}(\eta) \\
m(\eta ; s)=m_{0}+\sum_{n=1}^{\infty} s^{n} m_{n}(\eta) \\
n(\eta ; s)=n_{0}+\sum_{n=1}^{\infty} s^{n} n_{n}(\eta) \\
\theta(\eta ; s)=\theta_{0}+\sum_{n=1}^{\infty} s^{n} \theta_{n}(\eta) \\
f_{n}(\eta)=\left.\frac{1}{n !} \frac{\partial^{n} f(\eta ; s)}{\partial \eta^{n}}\right|_{s=0}, \quad g_{n}(\eta)=\left.\frac{1}{n !} \frac{\partial^{n} g(\eta ; s)}{\partial \eta^{n}}\right|_{s=0} \\
m_{n}(\eta)=\left.\frac{1}{n !} \frac{\partial^{n} m(\eta ; s)}{\partial \eta^{n}}\right|_{s=0}, \quad n_{n}(\eta)=\left.\frac{1}{n !} \frac{\partial^{n} n(\eta ; s)}{\partial \eta^{n}}\right|_{s=0} s^{n} \phi_{n}(\eta) \\
\theta_{n}(\eta)=\left.\frac{1}{n !} \frac{\partial^{n} \theta(\eta ; s)}{\partial \eta^{n}}\right|_{s=0}, \quad \phi_{n}(\eta)=\left.\frac{1}{n !} \frac{\partial^{n} \phi(\eta ; s)}{\partial \eta^{n}}\right|_{s=0}
\end{gathered}
$$

It can be noted that in the above series, convergence strongly depends upon $\hbar_{f}, \hbar_{g}$, $\hbar_{m}, \hbar_{n}, \hbar_{\theta}$ and $\hbar_{\phi}$.

Assuming that these nonzero auxiliary parameters are chosen so that equations converge at $s=1$, we thus obtain

$$
\begin{aligned}
& f(\eta)=f_{0}+\sum_{n=1}^{\infty} f_{n}(\eta) \\
& g(\eta)=g_{0}+\sum_{n=1}^{\infty} g_{n}(\eta) \\
& m(\eta)=m_{0}+\sum_{n=1}^{\infty} m_{n}(\eta) \\
& n(\eta)=n_{0}+\sum_{n=1}^{\infty} n_{n}(\eta) \\
& \theta(\eta)=\theta_{0}+\sum_{n=1}^{\infty} \theta_{n}(\eta)
\end{aligned}
$$




$$
\phi(\eta)=\phi_{0}+\sum_{n=1}^{\infty} \theta_{n}(\eta)
$$

Differentiating n-times Equations (40)-(45) with respect to $s$ and putting $s=0$, we have

$$
\begin{aligned}
£_{f}\left[f_{n}(\eta)-\chi_{n} f_{n-1}(\eta)\right] & =\hbar_{f} R_{f, n}(\eta) \\
£_{g}\left[g_{n}(\eta)-\chi_{n} g_{n-1}(\eta)\right] & =\hbar_{g} R_{g, n}(\eta) \\
£_{m}\left[m_{n}(\eta)-\chi_{n} m_{n-1}(\eta)\right] & =\hbar_{m} R_{m, n}(\eta) \\
£_{n}\left[n_{n}(\eta)-\chi_{n} n_{n-1}(\eta)\right] & =\hbar_{n} R_{n, n}(\eta) \\
£_{\theta}\left[\theta_{n}(\eta)-\chi_{n} \theta_{n-1}(\eta)\right] & =\hbar_{\theta} R_{\theta, n}(\eta) \\
£_{\phi}\left[\phi_{n}(\eta)-\chi_{n} \phi_{n-1}(\eta)\right] & =\hbar_{\phi} R_{\phi, n}(\eta)
\end{aligned}
$$

with the given boundary conditions,

$$
\begin{aligned}
& f_{n}(0)=0, \quad f_{n}^{\prime}(0)=0, \quad g_{n}(0)=1, \quad m_{n}(0)=0, \quad n_{n}(0)=0, \\
& \theta_{n}(0)=1, \quad \phi_{n}(0)=1 \\
& f_{n}(1)=0.5, \quad f_{n}^{\prime}(1)=0, \quad g_{n}(1)=S, \quad m_{n}(1)=1, \quad n_{n}(1)=1, \\
& \theta_{n}(1)=0, \quad \phi_{n}(1)=0 \\
& R_{f, n}(\eta)=f_{n-1}^{\prime \prime \prime \prime}(\eta)-S_{z}\left(3 f_{n-1}^{\prime \prime \prime}(\eta)+(\eta) f_{n-1}^{\prime \prime \prime}(\eta)-\right. \\
& \left.2 f_{n-1}^{\prime}(\eta) f_{n-1}^{\prime \prime}(\eta)-2 \sum_{j=0}^{n-1} f_{j}(\eta) f_{n-j-1}^{\prime \prime \prime}(\eta)\right)+2 S_{z} M_{z}^{2} \\
& \left(2 R _ { m } \sum _ { j = 0 } ^ { n - 1 } m _ { j } ( \eta ) \left[m_{n-j-1}^{\prime}(\eta)+\eta m_{n-j-1}^{\prime \prime}(\eta)+\right.\right. \\
& \left.\left.m_{n-j-1}(\eta) f_{n-j-1}^{\prime \prime}(\eta)\right]-m_{n-1}^{\prime}(\eta) m_{n-1}^{\prime \prime}(\eta)\right) \\
& R_{g, n}(\eta)=g_{n-1}^{\prime \prime}(\eta)-S_{z}\left(2 g_{n-1}(\eta)+(\eta) g_{n-1}^{\prime}(\eta)+2 \sum_{j=0}^{n-1}\right. \\
& {\left[g_{j}(\eta) f_{n-j-1}^{\prime}(\eta)-f_{j}(\eta) g_{n-j-1}^{\prime}(\eta)\right]+2 S_{z} M_{z} M_{y}} \\
& \sum_{j=0}^{n-1}\left(n_{j}(\eta) m_{n-j-1}^{\prime}(\eta)-n_{j(\eta)} m_{n-j-1}^{\prime}(\eta)\right) \\
& R_{m, n}(\eta)=m_{n-1}^{\prime \prime}(\eta)-R_{m}\left[m_{n-1}(\eta)+(\eta) m_{n-1}^{\prime}(\eta)+\right. \\
& \left.2 \sum_{j=0}^{n-1}\left(m_{j}(\eta) f_{n-j-1}^{\prime}(\eta)-f_{j}(\eta) m_{n-j-1}^{\prime}(\eta)\right)\right] \\
& R_{n, n}(\eta)=n_{n-1}^{\prime \prime}(\eta)-R_{m}\left[2 n_{n-1}(\eta)+(\eta) n_{n-1}^{\prime}(\eta)+\right. \\
& 2 \sum_{j=0}^{n-1}\left(n_{j}(\eta) f_{n-j-1}^{\prime}(\eta)-f_{j}(\eta) n_{n-j-1}^{\prime}(\eta)\right) \\
& \left.-2 \frac{M_{z}}{M_{y}}\left(m_{j}(\eta) g_{n-j-1}^{\prime}(\eta)-g_{j}(\eta) m_{n-j-1}^{\prime}(\eta)\right)\right] \\
& R_{\theta, n}(\eta)=\theta_{n-1}^{\prime \prime}(\eta)\left(3+4 R_{d}\right)+3 D_{u} P_{r n} \phi_{n-1}^{\prime \prime}(\eta)+ \\
& 3 S_{z} P_{r n} \sum_{j=0}^{n-1}\left(2 f_{j}(\eta) \theta_{n-j-1}^{\prime}(\eta)-\eta \theta_{n-j-1}^{\prime}(\eta)\right)
\end{aligned}
$$




$$
\begin{aligned}
& R_{\phi, n}(\eta)=\phi_{n-1}^{\prime \prime}(\eta)+S_{r t} S_{c h} \theta_{n-1}^{\prime \prime}(\eta)+ \\
& S_{z} S_{c h} \sum_{j=0}^{n-1}\left(2 f_{j}(\eta) \phi_{n-j-1}^{\prime}(\eta)-\eta \phi_{n-j-1}^{\prime}(\eta)\right)
\end{aligned}
$$

and $\chi_{n}=\{1$, if $n>1$, and 0 , if $n=1$.

Finally, the general solution can be written as

$$
\begin{gathered}
f_{n}(\eta)=\int_{0}^{\eta} \int_{0}^{\eta} \int_{0}^{\eta} \int_{0}^{\eta} \hbar_{f} R_{f, n}(z) d z d z d z d z+ \\
\chi_{n} f_{n-1}+K_{1 *} \eta^{3}+K_{2^{*}} \eta^{2}+K_{3^{*}} \eta+K_{4^{*}} \\
g_{n}(\eta)=\int_{0}^{\eta} \int_{0}^{\eta} \hbar_{g} R_{g, n}(z) d z d z+\chi_{n} g_{n-1}+q_{5^{*}}^{*} \eta+q_{6^{*}}^{*} \\
m_{n}(\eta)=\int_{0}^{\eta} \int_{0}^{\eta} \hbar_{m} R_{m, n}(z) d z d z+\chi_{n} m_{n-1}+q_{7^{*}}^{*} \eta+q_{8^{*}}^{*} \\
n_{n}(\eta)=\int_{0}^{\eta} \int_{0}^{\eta} \hbar_{n} R_{n, n}(z) d z d z+\chi_{n} n_{n-1}+q_{9^{*}}^{*} \eta+q_{10^{*}}^{*} \\
\theta_{n}(\eta)=\int_{0}^{\eta} \int_{0}^{\eta} \hbar_{\theta} R_{\theta, n}(z) d z d z+\chi_{n} \theta_{n-1}+q_{11^{*}}^{*} \eta+q_{12^{*}}^{*} \\
\phi_{n}(\eta)=\int_{0}^{\eta} \int_{0}^{\eta} \hbar_{\phi} R_{\phi, n}(z) d z d z+\chi_{n} \phi_{n-1}+q_{13^{*}}^{*} \eta+q_{14^{*}}^{*}
\end{gathered}
$$

thus, for $f(\eta), g(\eta), m(\eta), n(\eta), \theta(\eta)$ and $\phi(\eta)$, the exact solution becomes

$$
\begin{aligned}
& f(\eta) \approx \sum_{m=0}^{n} f_{m}(\eta) \\
& g(\eta) \approx \sum_{m=0}^{n} g_{m}(\eta) \\
& m(\eta) \approx \sum_{m=0}^{n} m_{m}(\eta) \\
& n(\eta) \approx \sum_{m=0}^{n} n_{m}(\eta) \\
& \theta(\eta) \approx \sum_{m=0}^{n} \theta_{m}(\eta) \\
& \phi(\eta) \approx \sum_{m=0}^{n} \phi_{m}(\eta)
\end{aligned}
$$

\section{Optimal Convergence Control Parameter}

It should be noted that the nonzero auxiliary parameters $\hbar_{f}, \hbar_{g}, \hbar_{m}, \hbar_{n}, \hbar_{\theta}$ and $\hbar_{\phi}$ are contained in the series solutions (59)-(64), through which the rate of the homotopy series solutions and convergence region can be determined. The average residual error was used to obtain the optimal values of $\hbar_{f}, \hbar_{g}, \hbar_{m}, \hbar_{n}, \hbar_{\theta}$ and $\hbar_{\phi}$ :

$$
\begin{aligned}
& \varepsilon_{n}^{f}=\frac{1}{L+1} \sum_{j=0}^{L}\left[\aleph_{f} *\left(\sum_{i=0}^{n} f *(\eta), \sum_{i=0}^{n} m *(\eta)\right)_{m=j \delta m}\right]^{2} d \eta \\
& \varepsilon_{n}^{m}=\frac{1}{L+1} \sum_{j=0}^{L}\left[\aleph_{m} *\left(\sum_{i=0}^{n} f *(\eta), \sum_{i=0}^{n} m *(\eta)\right)_{m=j \delta m}\right]^{2} d \eta
\end{aligned}
$$




$$
\begin{gathered}
\varepsilon_{n}^{g}=\frac{1}{L+1} \sum_{j=0}^{L}\left[\aleph _ { g } * \left(\sum_{i=0}^{n} f *(\eta), \sum_{i=0}^{n} g *(\eta), \sum_{i=0}^{n} m *(\eta),\right.\right. \\
\varepsilon_{n}^{n}=\frac{1}{L+1} \sum_{j=0}^{L}\left[\aleph _ { n } * \left(\sum_{i=0}^{n} f *(\eta), \sum_{i=0}^{n} g *(\eta), \sum_{i=0}^{n} m *(\eta),\right.\right. \\
\varepsilon_{n}^{\theta}=\frac{1}{L+1} \sum_{j=0}^{L}\left[\aleph _ { \theta } * \left(\sum_{i=0}^{n} f *(\eta), \sum_{i=0}^{n} \theta *(\eta),\right.\right. \\
\varepsilon_{n}^{\phi}=\frac{1}{L+1} \sum_{j=0}^{L}\left[\aleph_{\phi} *\left(\sum_{i=0}^{n} \phi *(\eta)\right)_{m=j}^{n} d \eta\right]^{2} d \eta \\
\left.\sum_{i=0}^{n} \phi *(\eta)\right) \\
\sum_{i=0}^{n} \theta *(\eta), \\
\sum_{m=j \delta m} d \eta
\end{gathered}
$$

Additionally,

$$
\varepsilon_{n}^{t}=\varepsilon_{n}^{f}+\varepsilon_{n}^{g}+\varepsilon_{n}^{m}+\varepsilon_{n}^{n}+\varepsilon_{n}^{\theta}+\varepsilon_{n}^{\phi}
$$

where the total squared residual error is $\varepsilon_{n}^{t}$. By applying Mathematica package BVPh 2.0 [40], we can minimize total average squared residual error. To acquire the local optimal convergence control parameters, the command Minimize was used.

\section{Results and Analysis}

Taking $10^{-40}$ as a maximum residual error, the problem is solved with the HAM BVPh 2.0 package. An investigation is made using 40th-order approximations. The provision of error analysis supports the authentication of results for many relevant physical parameters in Figure 2 and from the results given in Table 1.

Table 2 is provided to determine the equations' inaccuracy from momentum, magnetic field, energy and transportation equations. An increase in the order of approximation can be seen, and the solution obtained from these equations converges to exact analysis.

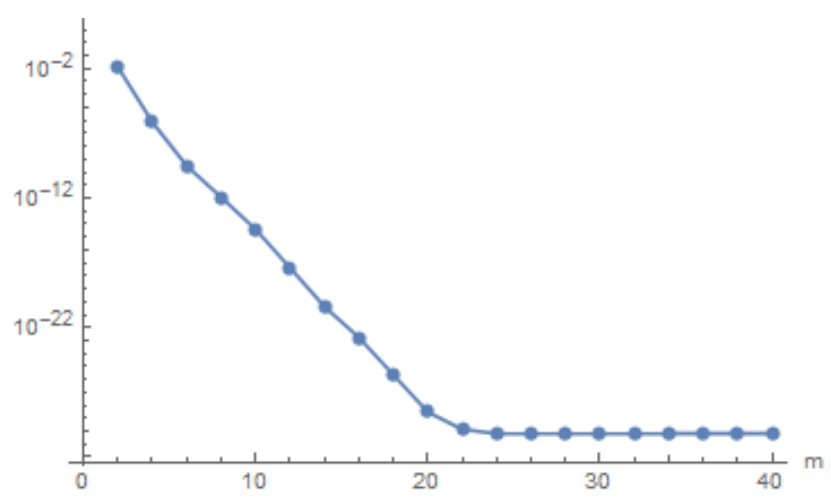

Figure 2. Total residual error with $S_{z}=-0.25, M_{x}=1, M_{y}=3, R_{m}=0.5, S=1, R_{d}=1, D=0.5$, $P_{r n}=0.05, S_{r t}=-0.25$ and $S_{c h}=0.5$.

The total residual error is visually shown in Figure 2 and numerically in Table 1 for different orders of approximation. It is seen that at the 30th order of approximation, the 
solution converges and the error nearly disappears. Table 2 illustrates the best convergence control parameter values for several approximation orders. From Table 3, we can observe the estimated values of velocity, magnetic field, concentration and temperature for different values of $\eta$. It also shows the solution is accurate by verification through the given boundary conditions of the problem (since the boundary conditions can be verified by observing the obtained numerical values at different points). The convergence of HAM solution for skin friction, magnetic flux, heat flux and mass flux for different orders of approximation is given in Table 4. It can be observed that the solution is convergent for fifth order of approximation. The analysis is carried out up to the 40th order of approximation.

Table 1. Estimating the total residual error for different orders of approximation.

\begin{tabular}{cccccc}
\hline$m$ & $\varepsilon_{m}^{f}$ & $\varepsilon_{m}^{g}$ & $\varepsilon_{m}^{m}$ & $\varepsilon_{m}^{n}$ \\
\hline 1 & 0.03811 & 0.00475 & 0.00066 & $\varepsilon_{m}^{\boldsymbol{\theta}}$ & 0.00088 \\
5 & $1.5 \times 10^{-9}$ & $1.0 \times 10^{-9}$ & $7.6 \times 10^{-12}$ & $2.1 \times 10^{-8}$ & $1.5 \times 10^{-15}$ \\
10 & $1.0 \times 10^{-18}$ & $9.5 \times 10^{-16}$ & $2.8 \times 10^{-21}$ & $2.7 \times 10^{-14}$ & $3.3 \times 10^{-17}$ \\
15 & $5.9 \times 10^{-28}$ & $1.0 \times 10^{-21}$ & $1.2 \times 10^{-30}$ & $4.1 \times 10^{-20}$ & $1.0 \times 10^{-26}$ \\
20 & $2.8 \times 10^{-30}$ & $1.3 \times 10^{-27}$ & $2.8 \times 10^{-33}$ & $6.5 \times 10^{-26}$ & $2.4 \times 10^{-32}$ \\
25 & $2.8 \times 10^{-30}$ & $1.8 \times 10^{-32}$ & $4.5 \times 10^{-33}$ & $1.6 \times 10^{-31}$ & $6.0 \times 10^{-35}$ \\
30 & $2.9 \times 10^{-30}$ & $1.2 \times 10^{-32}$ & $3.0 \times 10^{-33}$ & $1.5 \times 10^{-32}$ & $6.8 \times 10^{-34}$ \\
35 & $2.9 \times 10^{-30}$ & $1.2 \times 10^{-32}$ & $3.0 \times 10^{-33}$ & $1.5 \times 10^{-32}$ & $6.2 \times 10^{-35}$ \\
40 & $2.9 \times 10^{-30}$ & $1.2 \times 10^{-32}$ & $3.0 \times 10^{-33}$ & $1.5 \times 10^{-32}$ & $6.2 \times 10^{-34}$ \\
\hline
\end{tabular}

Table 2. Optimal values of convergence control parameters in comparison of different orders of approximation.

\begin{tabular}{cccccccc}
\hline Order & $\boldsymbol{h}_{\boldsymbol{f}}$ & $\boldsymbol{h}_{\boldsymbol{g}}$ & $\boldsymbol{h}_{\boldsymbol{m}}$ & $\boldsymbol{h}_{\boldsymbol{n}}$ & $\boldsymbol{h}_{\boldsymbol{\theta}}$ & $\boldsymbol{h}_{\boldsymbol{\phi}}$ & $\boldsymbol{\varepsilon}_{\boldsymbol{m}}^{\boldsymbol{t}}$ \\
\hline 2 & -1.0151 & -1.0459 & -0.8810 & -0.7341 & -0.1047 & -0.1040 \\
3 & -0.9471 & -1.1027 & -0.9423 & -0.8706 & -0.1110 & -1.0261 & $4.90 \times 10^{-6}$ \\
4 & -0.9678 & -1.0804 & -0.9360 & -0.8889 & -0.1053 & -0.9786 & $4.94 \times 10^{-8}$ \\
5 & -0.9290 & -1.0601 & -0.9433 & -0.9223 & -0.1140 & -1.0579 & $2.20 \times 10^{-10}$ \\
6 & -0.9583 & -1.0441 & -0.9435 & -0.9342 & -0.1369 & -1.0050 & $2.87 \times 10^{-12}$ \\
7 & -1.1097 & -1.0693 & -0.8652 & -0.9240 & -0.1172 & -1.0967 & $3.26 \times 10^{-12}$ \\
\hline
\end{tabular}

Table 3. Estimated values for velocity, magnetic field components, temperature and concentration in correspondance with different values of $\eta$.

\begin{tabular}{ccccccc}
\hline $\boldsymbol{\eta}$ & $f(\boldsymbol{\eta})$ & $\boldsymbol{g}(\boldsymbol{\eta})$ & $\boldsymbol{m}(\boldsymbol{\eta})$ & $\boldsymbol{n}(\boldsymbol{\eta})$ & $\boldsymbol{\theta}(\boldsymbol{\eta})$ & $\boldsymbol{\phi}(\boldsymbol{\eta})$ \\
\hline 0 & 0 & 1 & 0 & 0 & 1 & 1 \\
0.1 & 0.013945 & 1.038790 & 0.083334 & 0.090262 & 0.899994 & 0.899811 \\
0.2 & 0.051898 & 1.070930 & 0.167626 & 0.179204 & 0.799991 & 0.799707 \\
0.3 & 0.107937 & 1.095050 & 0.253977 & 0.268342 & 0.699991 & 0.699719 \\
0.4 & 0.176060 & 1.110120 & 0.343567 & 0.359189 & 0.599995 & 0.599833 \\
0.5 & 0.250219 & 1.115470 & 0.437564 & 0.453152 & 0.500000 & 0.500005 \\
0.6 & 0.324349 & 1.110810 & 0.537016 & 0.551440 & 0.400006 & 0.400177 \\
0.7 & 0.392389 & 1.096270 & 0.642747 & 0.654974 & 0.300009 & 0.300290 \\
0.8 & 0.448305 & 1.072300 & 0.755239 & 0.764292 & 0.200010 & 0.200299 \\
0.9 & 0.486125 & 1.039810 & 0.874512 & 0.879464 & 0.100006 & 0.100192 \\
1 & 0.5 & 1 & 1 & 1 & 0 & 0 \\
\hline
\end{tabular}


Table 4. The convergence of HAM solution for skin friction, magnetic flux, heat flux and mass flux.

\begin{tabular}{ccccccc}
\hline$m$ & $f^{\prime \prime}(\eta)$ & $-g^{\prime}(\eta)$ & $-m^{\prime}(\eta)$ & $-n^{\prime}(\eta)$ & $-\theta^{\prime}(\eta)$ & $-\phi^{\prime}(\eta)$ \\
\hline 1 & 2.9631400 & -0.3922459 & 0.8311292 & -0.9204676 & 1.0000650 & 1.0020067 \\
5 & 2.9804441 & -0.4157942 & -0.8318657 & -0.9141364 & 1.0000668 & 1.0020675 \\
10 & 2.9804441 & -0.4157877 & -0.8318658 & -0.9141418 & 1.0000668 & 1.002067 \\
15 & 2.9804441 & -0.4157877 & -0.8318658 & -0.9141418 & 1.0000668 & 1.002067 \\
20 & 2.9804441 & -0.4157877 & -0.8318658 & -0.9141418 & 1.0000668 & 1.002067 \\
25 & 2.9804441 & -0.4157877 & -0.8318658 & -0.9141418 & 1.0000668 & 1.002067 \\
30 & 2.9804441 & -0.4157877 & -0.8318658 & -0.9141418 & 1.0000668 & 1.002067 \\
35 & 2.9804441 & -0.4157877 & -0.8318658 & -0.9141418 & 1.0000668 & 1.002067 \\
40 & 2.9804441 & -0.4157877 & -0.8318658 & -0.9141418 & 1.0000668 & 1.002067 \\
\hline
\end{tabular}

In Tables 5-13, the numerical results for skin friction, magnetic flux, heat flux and mass flux are investigated. It is noted that increasing the squeeze parameter $S_{z}$ decreases $f^{\prime \prime}(0)$, $-n^{\prime}(0)-\theta^{\prime}(0)$ and $-\phi^{\prime}(0)$, while oppositely $-g^{\prime}(0)$ and $-m^{\prime}(0)$ increases with increasing $S_{z}$. Increasing the values of $M_{y}$ shows a certain increasing effect on both $-g^{\prime}(0)$ and $-n^{\prime}(0)$ while the effect on $f^{\prime \prime}(0),-m^{\prime}(0),-\theta^{\prime}(0)$ and $-\phi^{\prime}(0)$ is negligible, as can be seen through Table 6. Similarly, for increasing $M x$ there is a gradual increase in the values of $f^{\prime \prime}(0)$, $-g^{\prime}(0)$ and $-m^{\prime}(0)$ while the values of $-n^{\prime}(0),-\theta^{\prime}(0)$ and $-\phi^{\prime}(0)$ show gradual decreases, as shown in Table 7.

Table 5. Effect of $S_{z}$ on skin friction, magnetic flux, heat flux and mass flux.

\begin{tabular}{ccccccc}
\hline$S_{z}$ & $f^{\prime \prime}(\eta)$ & $-g^{\prime}(\eta)$ & $-m^{\prime}(\eta)$ & $-n^{\prime}(\eta)$ & $-\theta^{\prime}(\eta)$ & $-\phi^{\prime}(\eta)$ \\
\hline-0.01 & 2.999403 & -0.017073 & -0.831885 & -0.569941 & 0.999976 & 0.999247 \\
-0.25 & 2.985167 & -0.467599 & -0.831912 & -0.553833 & 0.999397 & 0.981150 \\
-0.75 & 2.956173 & -1.768705 & -0.831980 & -0.505905 & 0.998187 & 0.943287 \\
-1.25 & 2.928189 & -4.069057 & -0.832065 & -0.418285 & 0.996968 & 0.905179 \\
\hline
\end{tabular}

Table 6. Effect of $M_{y}$ on skin friction, magnetic flux, heat flux and mass flux.

\begin{tabular}{ccccccc}
\hline $\boldsymbol{M}_{\boldsymbol{y}}$ & $\boldsymbol{f}^{\prime \prime}(\eta)$ & $-\boldsymbol{g}^{\prime}(\eta)$ & $-\boldsymbol{m}^{\prime}(\eta)$ & $-\boldsymbol{n}^{\prime}(\eta)$ & $-\boldsymbol{\theta}^{\prime}(\eta)$ & $-\boldsymbol{\phi}^{\prime}(\eta)$ \\
\hline 1 & 2.985167 & -0.457030 & -0.831912 & -0.121005 & 0.999397 & 0.981150 \\
3 & 2.985167 & -0.467600 & -0.831912 & -0.553832 & 0.999397 & 0.981150 \\
5 & 2.985167 & -0.478166 & -0.831912 & -0.640398 & 0.999397 & 0.981150 \\
7 & 2.985167 & -0.488735 & -0.831912 & -0.677498 & 0.999397 & 0.981150 \\
\hline
\end{tabular}

Table 7. Effect of $M_{z}$ on skin friction, magnetic flux, heat flux and mass flux.

\begin{tabular}{ccccccc}
\hline $\boldsymbol{M}_{\boldsymbol{z}}$ & $\boldsymbol{f}^{\prime \prime}(\eta)$ & $-\boldsymbol{g}^{\prime}(\eta)$ & $-\boldsymbol{m}^{\prime}(\eta)$ & $-\boldsymbol{n}^{\prime}(\eta)$ & $-\boldsymbol{\theta}^{\prime}(\eta)$ & $-\boldsymbol{\phi}^{\prime}(\eta)$ \\
\hline 1 & 2.980444 & -0.415788 & -0.831866 & -0.914141 & 0.999398 & 0.981167 \\
1.5 & 2.985167 & -0.435894 & -0.831912 & -0.986660 & 0.999397 & 0.981150 \\
2 & 2.991858 & -0.466561 & -0.831978 & -1.060309 & 0.999397 & 0.981125 \\
2.5 & 3.000600 & -0.508235 & -0.832064 & -1.135544 & 0.999396 & 0.981093 \\
\hline
\end{tabular}

Further, Table 8 depicts the impact of magnetic Reynold number on skin friction showing that with increase in the values of $R_{m}$, the values of $-g^{\prime}(0)$ increases gradually, but on the other hand, $f^{\prime \prime}(0),-m^{\prime}(0),-n^{\prime}(0)$ and $-\phi^{\prime}(0)$ decreases, and the effect on $-\theta^{\prime}(0)$ is negligible. Similarly, from Table 9, it can be seen that the Dufour number has a direct effect on both $-\theta^{\prime}(0)$ and $-\phi^{\prime}(0)$. The effect of Radiation Parameter $R_{d}$ can be depicted through Table 10, showing that $-\theta^{\prime}(0)$ and $-\phi^{\prime}(0)$ increases with increasing $R_{d}$, while on the other hand, the effect on $f^{\prime \prime}(0),-g^{\prime}(0),-m^{\prime}(0)$ and $-n^{\prime}(0)$ is negligible.

Table 11 depicts the impact of Prandtl number on skin friction, showing that with increase in the values of $P_{r n}$, the values of $-\theta^{\prime}(0)$ and $-\phi^{\prime}(0)$ decrease gradually. The 
effect of $S_{r t}$ number from Table 12 depicts that $-\theta^{\prime}(0)$ decreases while $-\phi^{\prime}(0)$ increases by increasing the values of $S_{r t}$. The effect of Schmidt number $S_{c h}$ can be seen through Table 13. It can be observed that the $S_{c h}$ number has a direct effect $-\theta^{\prime}(0)$ and inverse effect on $-\phi^{\prime}(0)$. Furthermore, Figures 3-5 represent the error profile for velocity $(f(\eta)$ and $g(\eta))$ and magnetic field components, -i.e., $m(\eta)$ and $n(\eta)$, as well as temperature and concentration, i.e., $\theta(\eta)$ and $\phi(\eta)$, respectively. A three-dimensional profile for the velocity, magnetic field, concentration and temperature distribution is also represented in Figures 6-8, showing that the flow variables satisfy the given boundary conditions. The impacts of several related flow parameters on velocity and magnetic field components are visually depicted.
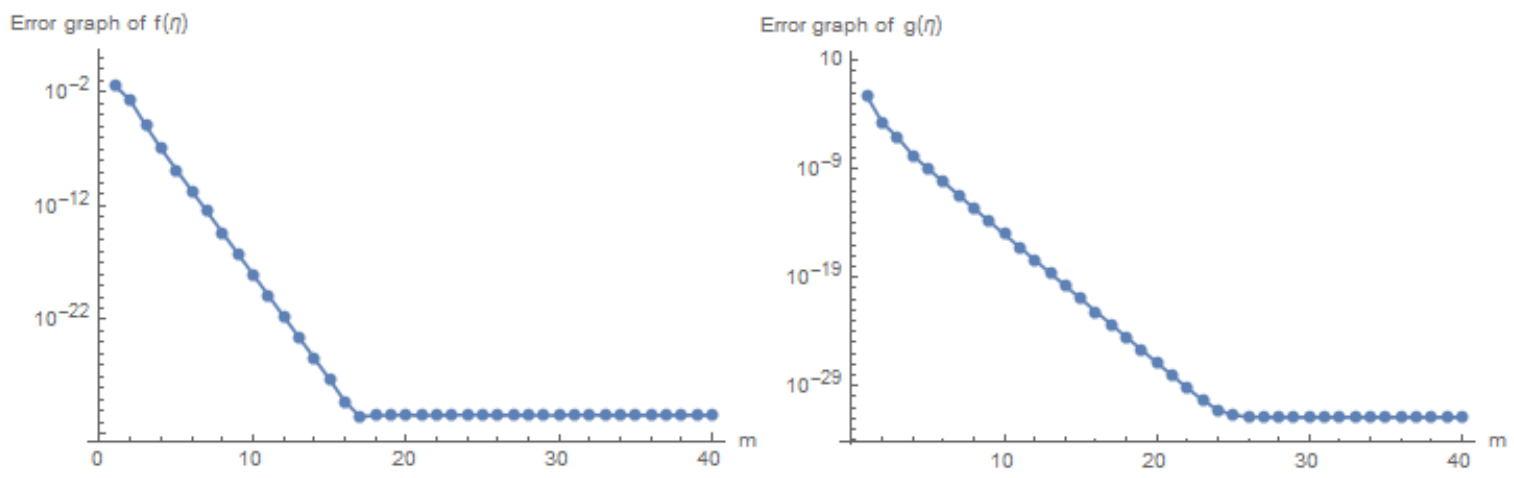

Figure 3. Error profile for $f(\eta)$ and $g(\eta)$ with $S_{z}=-0.25, M_{x}=1, M_{y}=3, R_{m}=0.5, S=1, R_{d}=1, D=0.5, P_{r n}=0.05$, $S_{r t}=-0.25$ and $S_{c h}=0.5$.
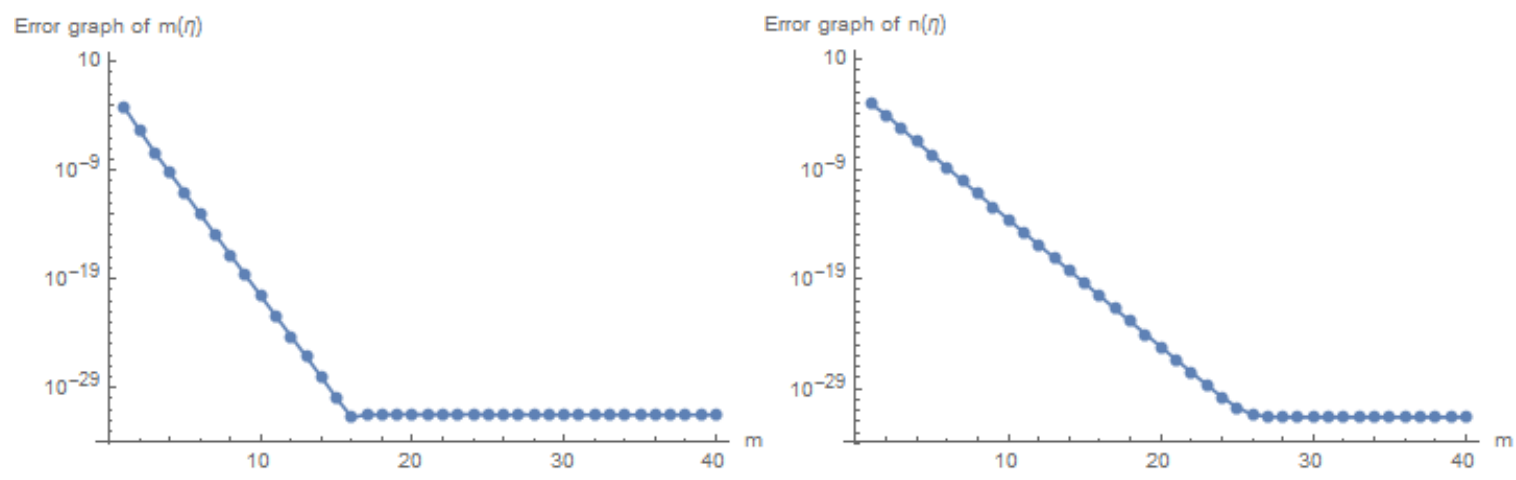

Figure 4. Error profile for $m(\eta)$ and $n(\eta)$ with $S_{z}=-0.25, M_{x}=1, M_{y}=3, R_{m}=0.5, S=1, R_{d}=1, D=0.5, P_{r n}=0.05$, $S_{r t}=-0.25$ and $S_{c h}=0.5$.
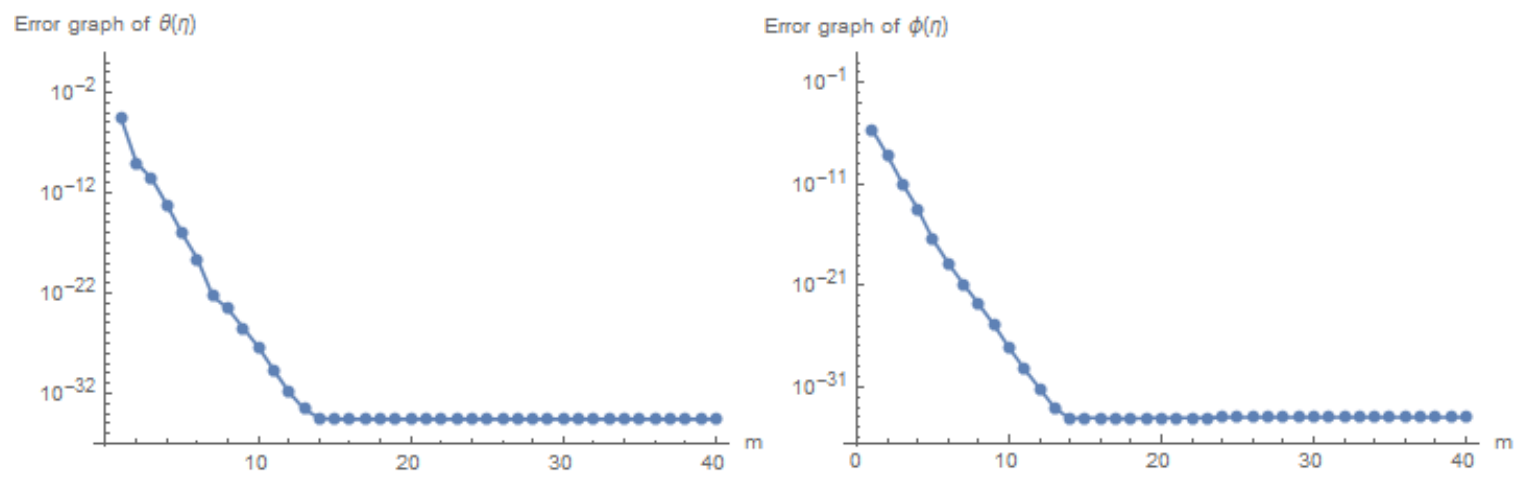

Figure 5. Error profile for $\theta(\eta)$ and $\phi(\eta)$ with $S_{z}=-0.25, M_{x}=1, M_{y}=3, R_{m}=0.5, S=1, R_{d}=1, D=0.5, P_{r n}=0.05$, $S_{r t}=-0.25$ and $S_{c h}=0.5$. 
Table 8. Effect of $R_{m}$ on skin friction, magnetic flux, heat flux and mass flux.

\begin{tabular}{ccccccc}
\hline $\boldsymbol{R}_{\boldsymbol{m}}$ & $f^{\prime \prime}(\eta)$ & $-\boldsymbol{g}^{\prime}(\eta)$ & $-\boldsymbol{m}^{\prime}(\eta)$ & $-\boldsymbol{n}^{\prime}(\eta)$ & $-\boldsymbol{\theta}^{\prime}(\eta)$ & $-\boldsymbol{\phi}^{\prime}(\eta)$ \\
\hline 0.1 & 2.976998 & -0.408251 & -0.962689 & -0.921462 & 0.998762 & 0.981100 \\
0.5 & 2.977633 & -0.414660 & -0.831838 & -0.663347 & 0.998762 & 0.981098 \\
1 & 2.977560 & -0.419649 & -0.701154 & -0.433268 & 0.998762 & 0.981097 \\
1.5 & 2.976902 & -0.422670 & -0.597416 & -0.271630 & 0.998762 & 0.981099 \\
\hline
\end{tabular}

Table 9. Effect of $D_{u}$ on skin friction, magnetic flux, heat flux and mass flux.

\begin{tabular}{ccccccc}
\hline $\boldsymbol{D}_{\boldsymbol{u}}$ & $f^{\prime \prime}(\eta)$ & $-g^{\prime}(\eta)$ & $-\boldsymbol{m}^{\prime}(\eta)$ & $-\boldsymbol{n}^{\prime}(\eta)$ & $-\boldsymbol{\theta}^{\prime}(\eta)$ & $-\boldsymbol{\phi}^{\prime}(\eta)$ \\
\hline 0.1 & 2.985167 & -0.467599 & -0.831912 & -0.553833 & 0.999236 & 0.981129 \\
0.75 & 2.985167 & -0.467599 & -0.831912 & -0.553833 & 0.999498 & 0.981162 \\
1.25 & 2.985167 & -0.467599 & -0.831912 & -0.553833 & 0.999699 & 0.981188 \\
1.75 & 2.985167 & -0.467599 & -0.831912 & -0.553833 & 0.999900 & 0.981213 \\
\hline
\end{tabular}

Table 10. Effect of $R_{d}$ on skin friction, magnetic flux, heat flux and mass flux.

\begin{tabular}{ccccccc}
\hline $\boldsymbol{R}_{\boldsymbol{d}}$ & $f^{\prime \prime}(\eta)$ & $-g^{\prime}(\eta)$ & $-\boldsymbol{m}^{\prime}(\eta)$ & $-n^{\prime}(\eta)$ & $-\boldsymbol{\theta}^{\prime}(\eta)$ & $-\boldsymbol{\phi}^{\prime}(\eta)$ \\
\hline 0.1 & 2.977633 & -0.414660 & -0.831838 & -0.663347 & 0.998762 & 0.981098 \\
0.5 & 2.977633 & -0.414660 & -0.831838 & -0.663347 & 0.999158 & 0.981147 \\
1 & 2.977633 & -0.414660 & -0.831838 & -0.663347 & 0.999398 & 0.981177 \\
1.5 & 2.977633 & -0.414660 & -0.831838 & -0.663347 & 0.999532 & 0.981194 \\
\hline
\end{tabular}

Table 11. Effect of $P_{r n}$ on skin friction, magnetic flux, heat flux and mass flux.

\begin{tabular}{ccccccc}
\hline $\boldsymbol{P}_{\boldsymbol{r}}$ & $f^{\prime \prime}(\eta)$ & $-\boldsymbol{g}^{\prime}(\eta)$ & $-\boldsymbol{m}^{\prime}(\eta)$ & $-\boldsymbol{n}^{\prime}(\eta)$ & $-\boldsymbol{\theta}^{\prime}(\eta)$ & $-\boldsymbol{\phi}^{\prime}(\eta)$ \\
\hline 0.05 & 2.977633 & -0.414660 & -0.831838 & -0.663347 & 0.999532 & 0.981194 \\
0.1 & 2.977633 & -0.414660 & -0.831838 & -0.663347 & 0.999064 & 0.981135 \\
0.25 & 2.977633 & -0.414660 & -0.831838 & -0.663347 & 0.997668 & 0.980960 \\
0.5 & 2.977633 & -0.414660 & -0.831838 & -0.663347 & 0.995361 & 0.980671 \\
\hline
\end{tabular}

Table 12. Effect of $S_{r t}$ on skin friction, magnetic flux, heat flux and mass flux.

\begin{tabular}{ccccccc}
\hline$S_{r t}$ & $f^{\prime \prime}(\eta)$ & $-g^{\prime}(\eta)$ & $-m^{\prime}(\eta)$ & $-n^{\prime}(\eta)$ & $-\theta^{\prime}(\eta)$ & $-\phi^{\prime}(\eta)$ \\
\hline 0.1 & 3.000040 & -0.222019 & -0.831954 & -0.622051 & 0.999758 & 0.992499 \\
0.5 & 3.000040 & -0.222019 & -0.831954 & -0.622051 & 0.999758 & 0.992548 \\
1 & 3.000040 & -0.222019 & -0.831954 & -0.622051 & 0.999758 & 0.992609 \\
1.5 & 3.000040 & -0.222019 & -0.831954 & -0.622051 & 0.999757 & 0.992670 \\
\hline
\end{tabular}

Table 13. Effect of $S_{c h}$ on skin friction, magnetic flux, heat flux and mass flux.

\begin{tabular}{ccccccc}
\hline$S_{c h}$ & $f^{\prime \prime}(\eta)$ & $-g^{\prime}(\eta)$ & $-m^{\prime}(\eta)$ & $-n^{\prime}(\eta)$ & $-\theta^{\prime}(\eta)$ & $-\phi^{\prime}(\eta)$ \\
\hline 0.5 & 2.985167 & -0.478167 & -0.831912 & -0.640398 & 0.999397 & 0.981150 \\
1 & 2.985167 & -0.478167 & -0.831912 & -0.640398 & 0.999598 & 0.962350 \\
1.5 & 2.985167 & -0.478167 & -0.831912 & -0.640398 & 0.999799 & 0.943600 \\
2 & 2.985167 & -0.478167 & -0.831912 & -0.640398 & 1.000000 & 0.924901 \\
\hline
\end{tabular}



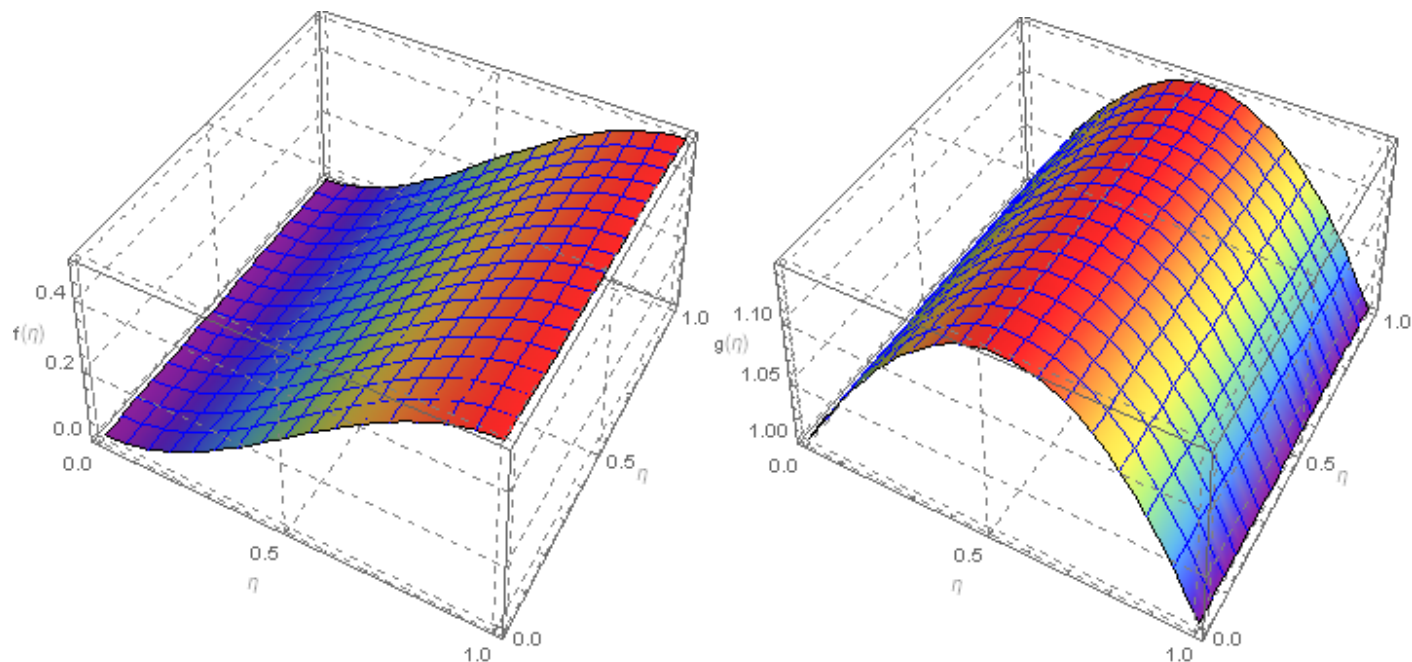

Figure 6. 3D graph for $f(\eta)$ and $g(\eta)$ with $S_{z}=-0.25, M_{x}=1, M_{y}=3, R_{m}=0.5, S=1, R_{d}=1, D=0.5, P_{r n}=0.05$, $S_{r t}=-0.25$ and $S_{c h}=0.5$.
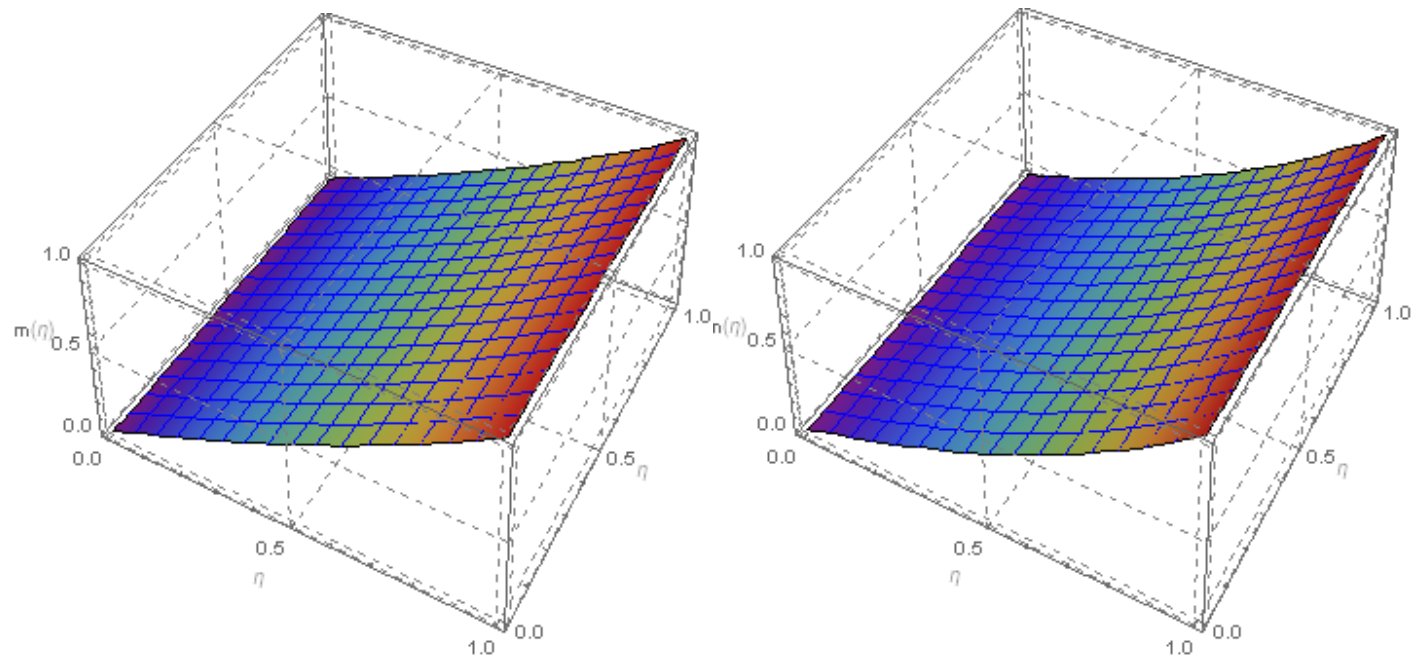

Figure 7. 3D graph for $m(\eta)$ and $n(\eta)$ with $S_{z}=-0.25, M_{x}=1, M_{y}=3, R_{m}=0.5, S=1, R_{d}=1, D=0.5, P_{r n}=0.05$, $S_{r t}=-0.25$ and $S_{c h}=0.5$.
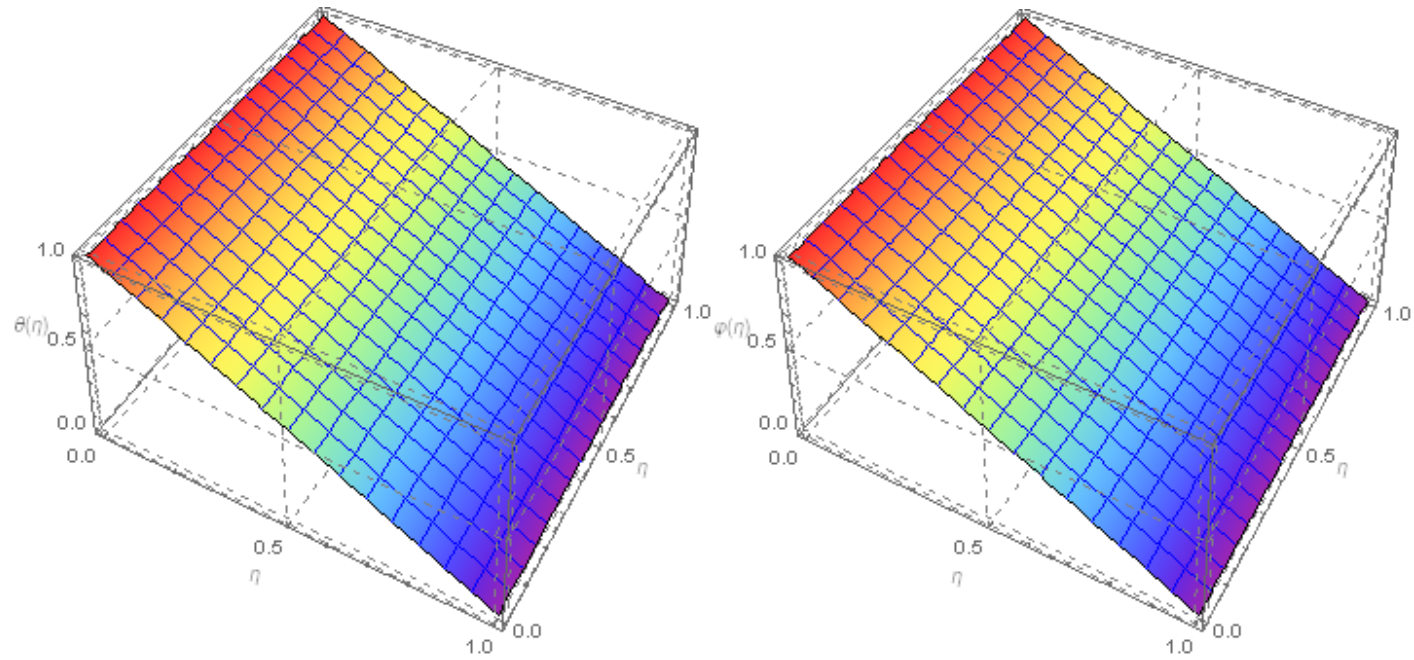

Figure 8. 3D graph for $\theta(\eta)$ and $\phi(\eta)$ with $S_{z}=-0.25, M_{x}=1, M_{y}=3, R_{m}=0.5, S=1, R_{d}=1, D=0.5, P_{r n}=0.05$, $S_{r t}=-0.25$ and $S_{c h}=0.5$. 


\section{Discussion}

In this section, the effects of different involved flow parameters are discussed graphically on velocity and magnetic field components. The effect of squeezing Reynold numbers can be seen in Figures 9-12. It is observed that for fix values of the other parameters, i.e., $M_{x}, M_{y}, R_{m}, S, R_{d}, D, P_{r n}, S_{r t}$ and $S_{c h}$, it is clear that increasing the squeeze Reynold number (moving upper disc towards lower disc with increasing order pattern) has a direct effect on the velocity components in both the $y$ - and z-direction. On the other hand, in the $x$-direction, the velocity increases initially but shows a decreasing effect as $\eta \rightarrow 1$, where, as in the case of magnetic field, the increase in squeeze Reynold number results in a decrease in magnetic field component along the z-direction, while a direct relation is observed for the y-component of the magnetic field, i.e., increasing the squeeze number causes an increase in the magnetic field along the y-direction. On both concentration and temperature distribution, the direct effect of the squeeze number is observed.
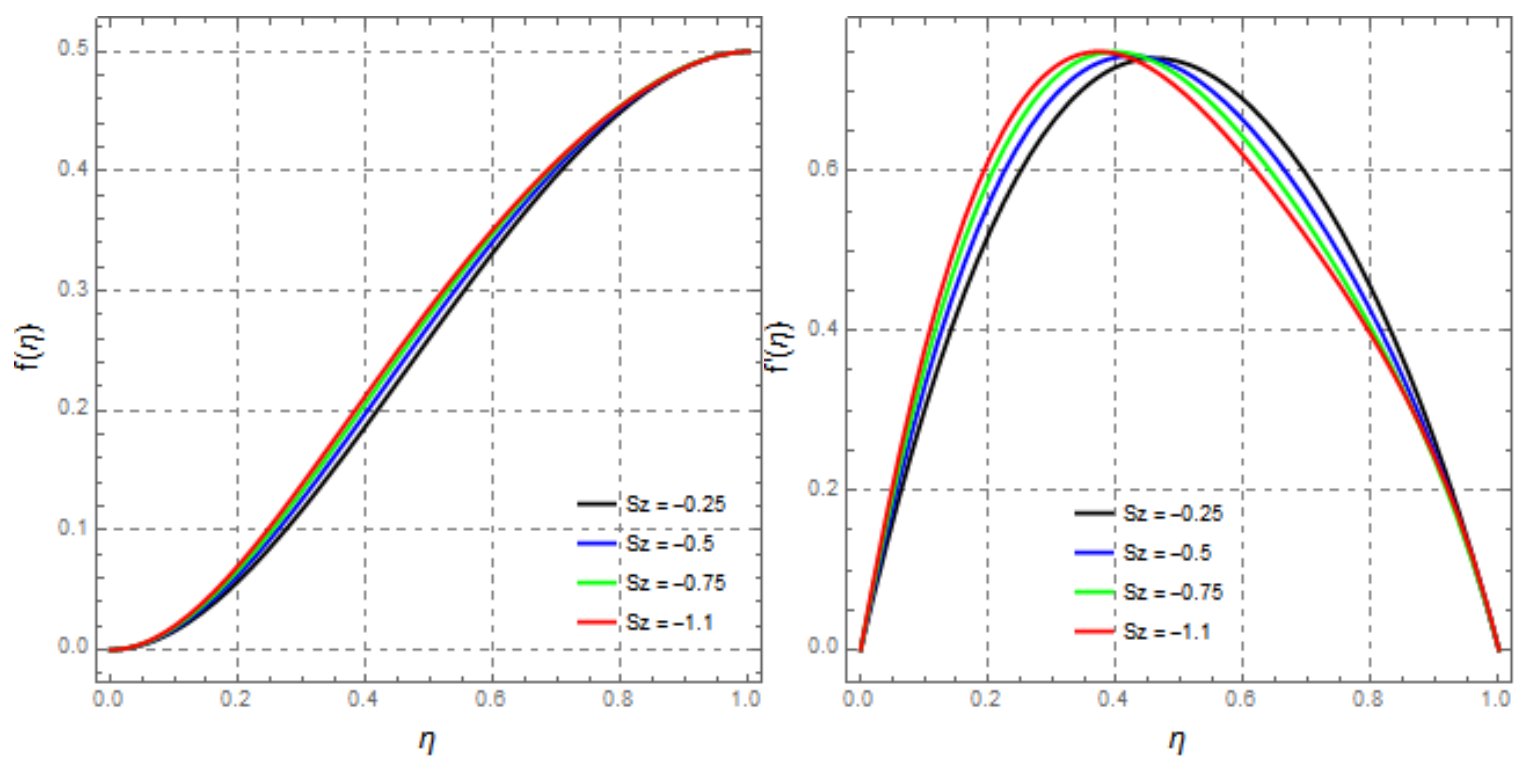

Figure 9. Impact of squeeze Reynold number $S_{z}$ on $f(\eta)$ and $f^{\prime}(\eta)$, keeping $M_{z}=-3.25, M_{y}=7, R_{m}=-2, S=1, R_{d}=0.5$, $D_{u}=0.1, P_{r n}=2, S_{r t}=0.2$ and $S_{c h}=1$.
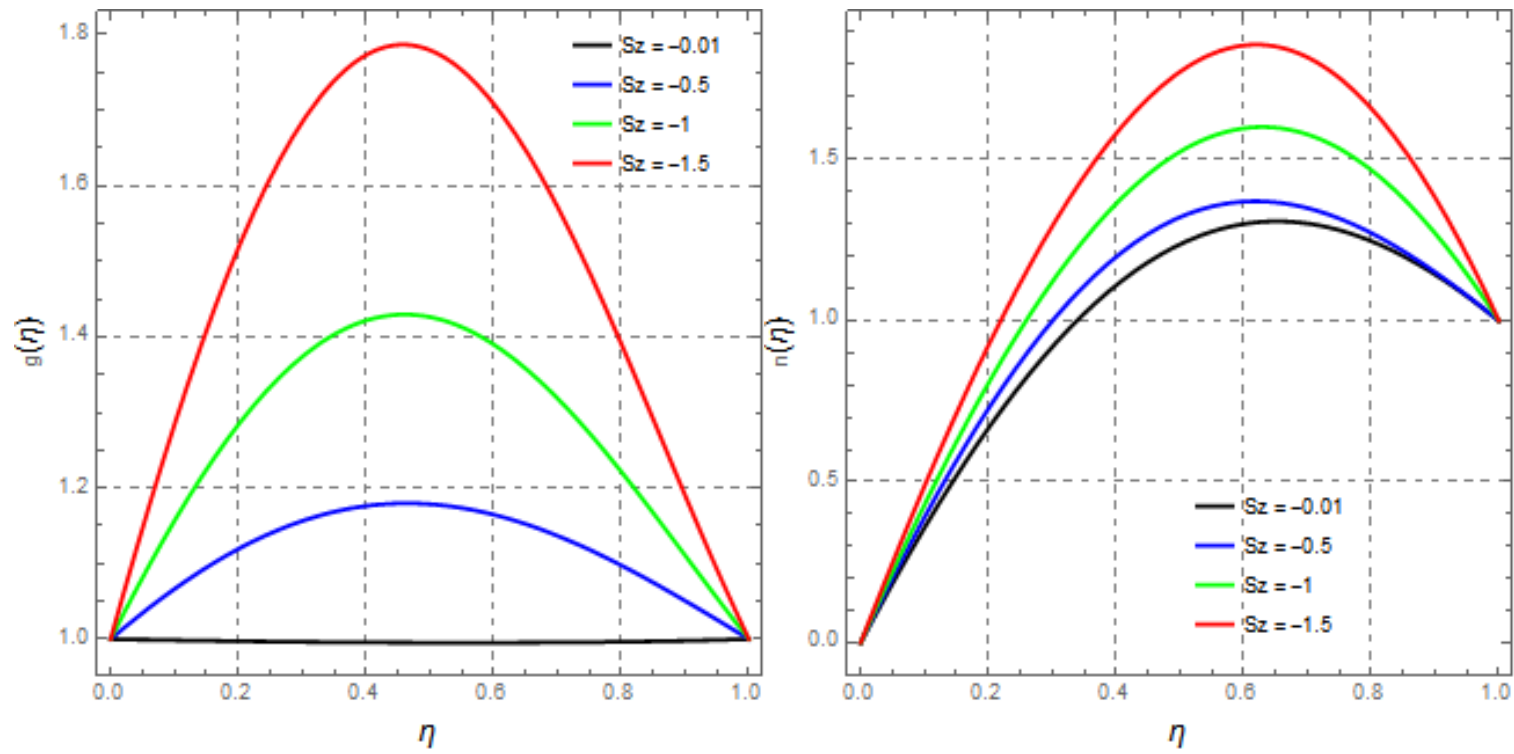

Figure 10. Observing the effect of squeeze Reynold number $S_{z}$ on $g(\eta)$ and $n(\eta)$ with $M_{z}=-1, M_{y}=0.5, R_{m}=-0.75$, $S=1, R_{d}=0.5, D_{u}=0.1, P_{r n}=5, S_{r t}=0.2$ and $S_{c h}=1$. 


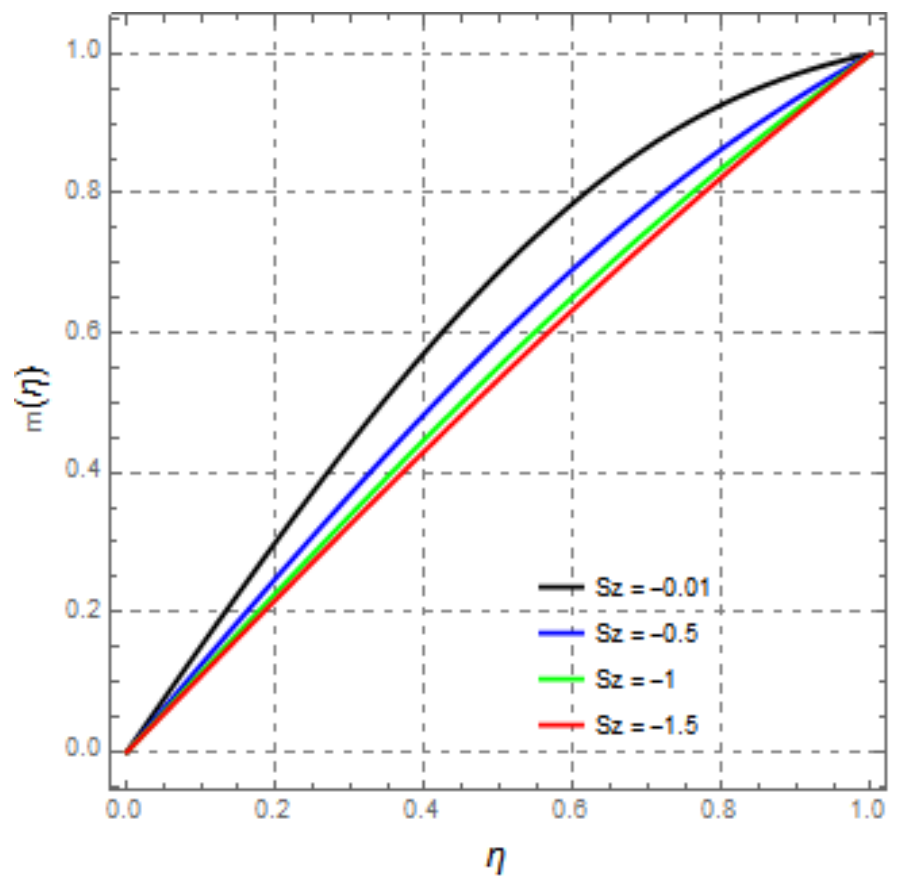

Figure 11. Impact of squeeze Reynold number $S_{z}$ on $m(\eta)$, keeping $M_{z}=-1.5, M_{y}=0.5, R_{m}=-1$, $S=1, R_{d}=0.5, D_{u}=0.1, P_{r n}=2, S_{r t}=0.2$ and $S_{c h}=1$.
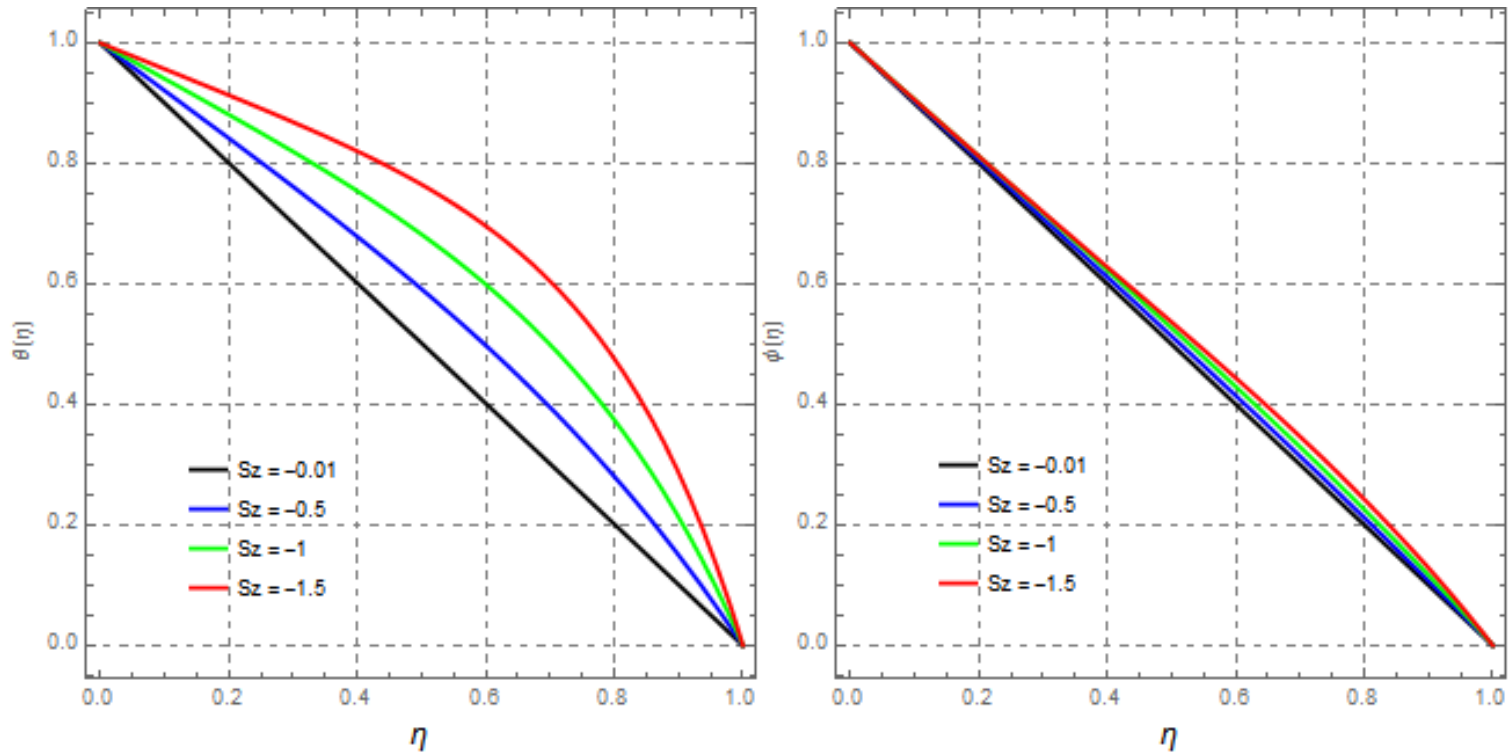

Figure 12. Observing the effect of squeeze Reynold number $S_{z}$ on $\theta(\eta)$ and $\phi(\eta)$ with $M_{z}=-1, M_{y}=0.5, R_{m}=-0.75$, $S=1, R_{d}=0.5, D_{u}=0.1, P_{r n}=5, S_{r t}=0.2$ and $S_{c h}=1$.

The magnetic field strength $M_{z}$ is the magnetic field's dimensionless axial strength. For additional parameters with fixed values, from Figures 13-16, it can be seen that increasing $M_{z}$ generates a decrease in the velocity component $f(\eta)$, whereas decreasing the magnetic field strength along the x-component causes a rise in the velocity $g(\eta)$. The initial velocity along the $\mathrm{x}$-component decreases with increasing magnetic field intensity for the velocity component $f^{\prime}(\eta)$ but starts to increase as $\eta \rightarrow 1$. The figure also shows how $M_{z}$ has an increasing influence on the magnetic field component $m(\eta)$ and $n(\eta)$. When discussing temperature distribution, increasing $M_{z}$ causes $\theta(\eta)$ to increase as well, whereas decreasing $\phi(\eta)$ causes $p h i(\eta)$ to drop. Figures 17 and 18 show the effect of magnetic field intensity $M_{y}$, which is the strength of the magnetic field in the y-direction. It is discovered that as 
the magnetic field intensity increases, both velocity $g(\eta)$ and the magnetic field component $n(\eta)$ drop, indicating an inverse relationship in both cases. To observe the effect of magnetic Reynold number $R_{m}$, Figures 19-22 illustrate the relations, showing that an increases in the magnetic Reynold number causes a decrease in the velocity component $f(\eta)$ with fixed values of other parameters, but increases in the velocity component $g(\eta)$ with fixed values of other parameters results in an increase in the value of shear force due to distribution of body force in a non-uniform manner. Body force accelerates near the relative core wall layer because Lorentz force is small near the squeezed plate (because of the current being almost parallel to the magnetic field).
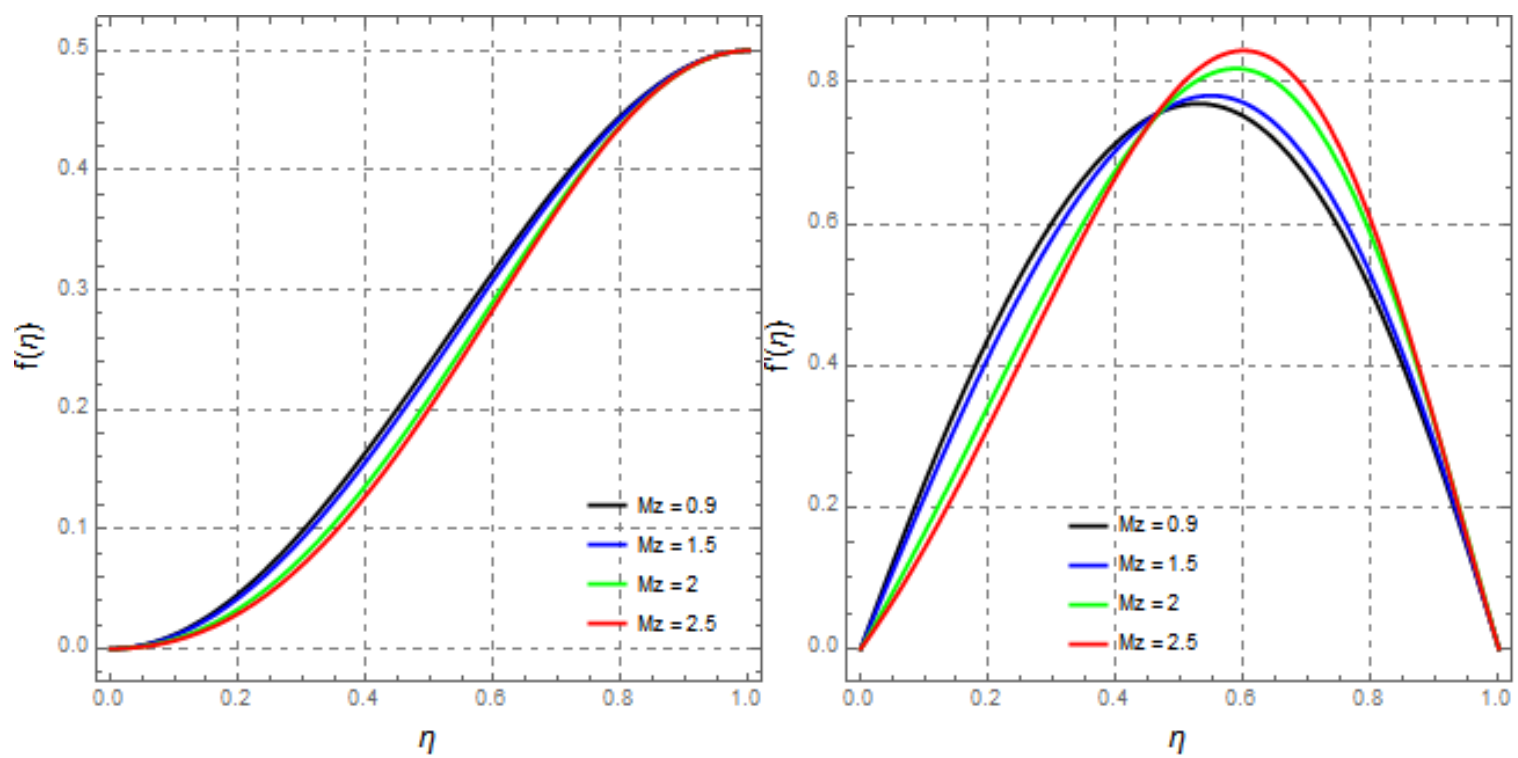

Figure 13. Observing the effect of magnetic strength parameter $M_{z}$ on $f(\eta)$ and $f^{\prime}(\eta)$ with $S_{z}=2, M_{y}=3, R_{m}=1, S=1$, $R_{d}=1, D_{u}=0.1, P_{r n}=1, S_{r t}=0.2$ and $S_{c h}=1$.
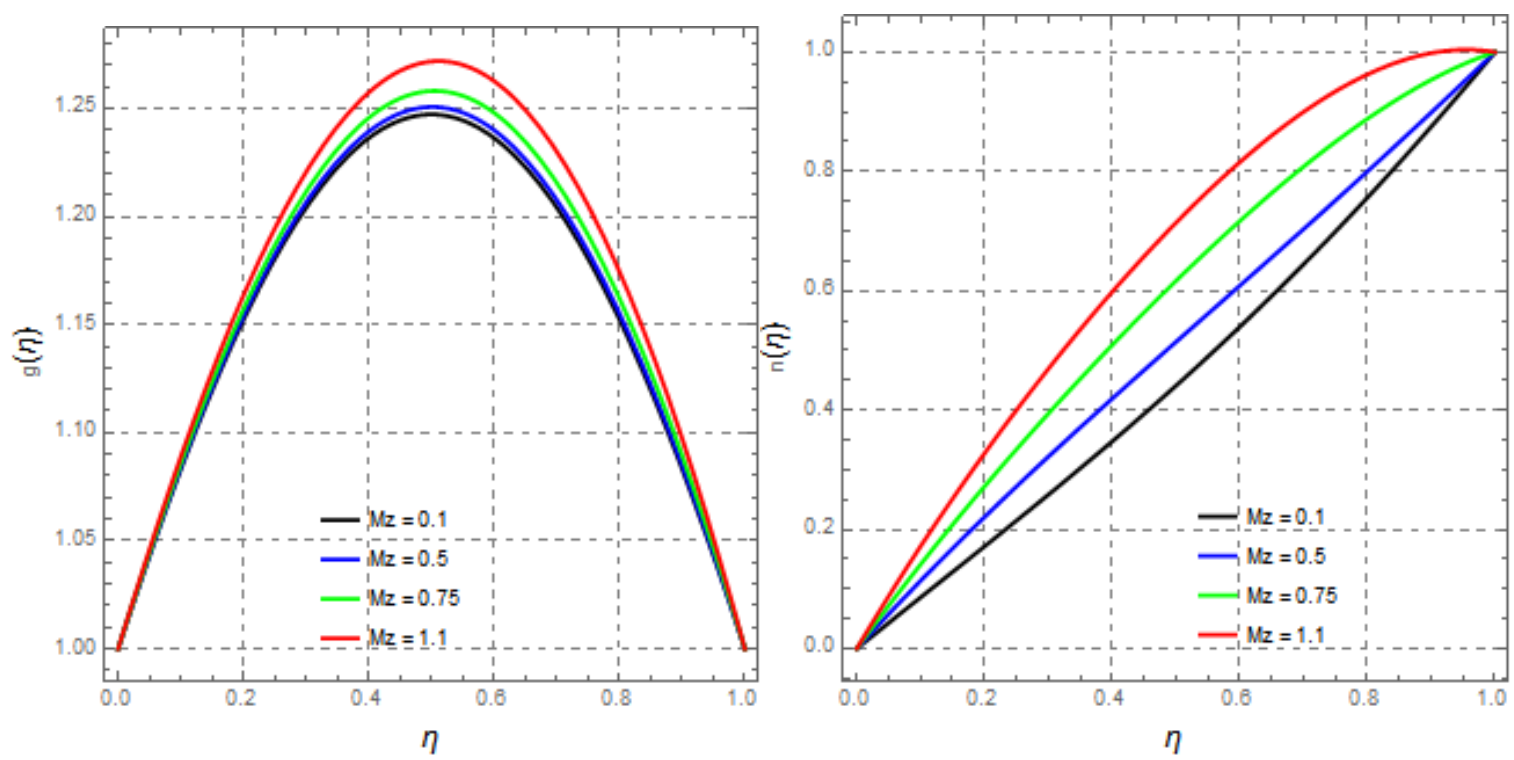

Figure 14. Observing the effect of magnetic strength parameter $M_{z}$ on $g(\eta)$ and $n(\eta)$ with $S_{z}=-0.25, M_{y}=3, R_{m}=1$, $S=1, R_{d}=0.5, D_{u}=0.1, P_{r n}=1, S_{r t}=2$ and $S_{c h}=0.5$. 


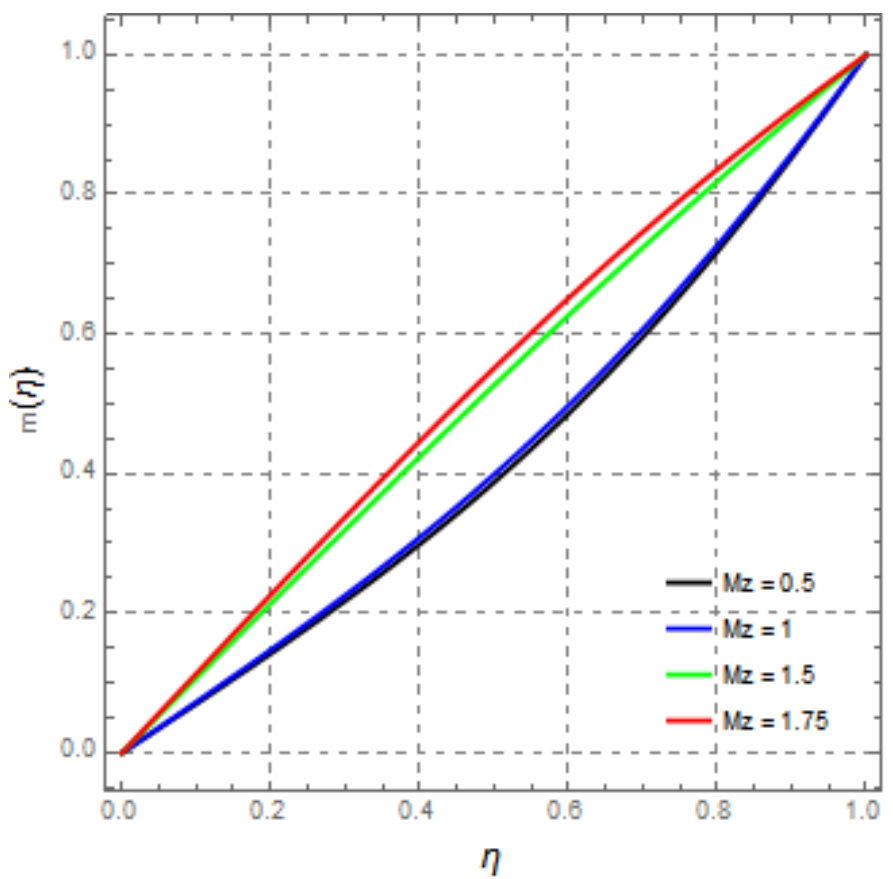

Figure 15. Observing the effect of magnetic strength parameter $M_{z}$ on $m(\eta)$ with $S_{z}=-0.25, M_{y}=3$, $R_{m}=1, S=1, R_{d}=1, D_{u}=0.1, P_{r n}=1, S_{r t}=2$ and $S_{c h}=0.5$.
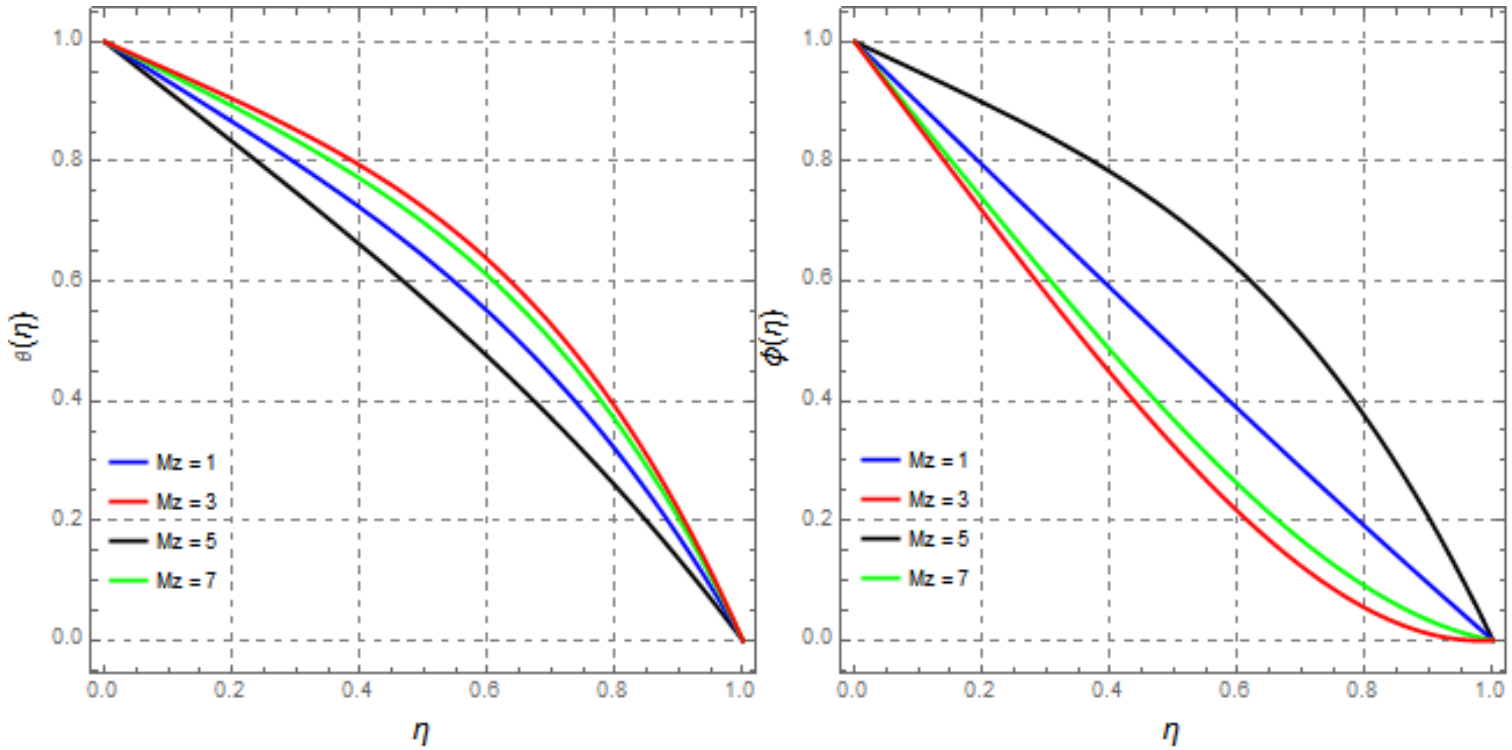

Figure 16. Observing the effect of magnetic strength parameter $M_{z}$ on $\theta(\eta)$ and $\phi(\eta)$ with $S_{z}=-1.5, M_{y}=1, R_{m}=-1$, $S=1, R_{d}=-1, D_{u}=0.1, P_{r n}=-1, S_{r t}=-2$ and $S_{c h}=-0.5$. 


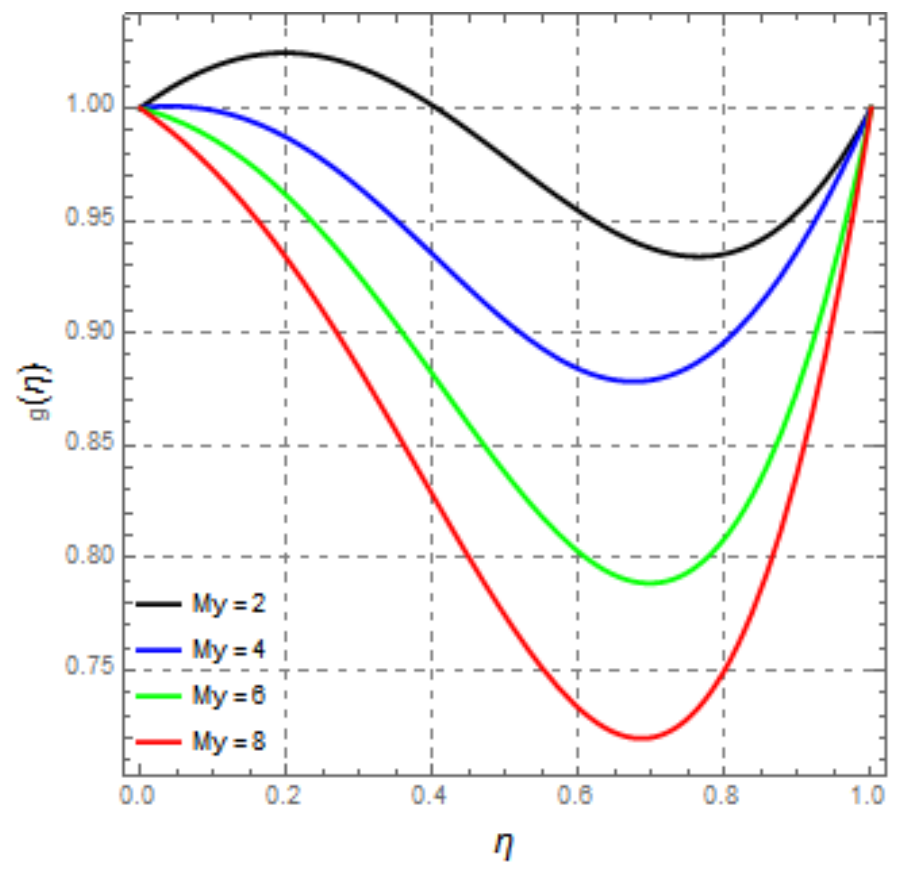

Figure 17. Observing the effect of magnetic strength parameter $M_{y}$ on $g(\eta)$ with $S_{z}=-0.5, M_{z}=$ $-0.5, R_{m}=-2, S=1, R_{d}=0.5, D_{u}=0.1, P_{r n}=5, S_{r t}=0.2$ and $S_{c h}=1$.

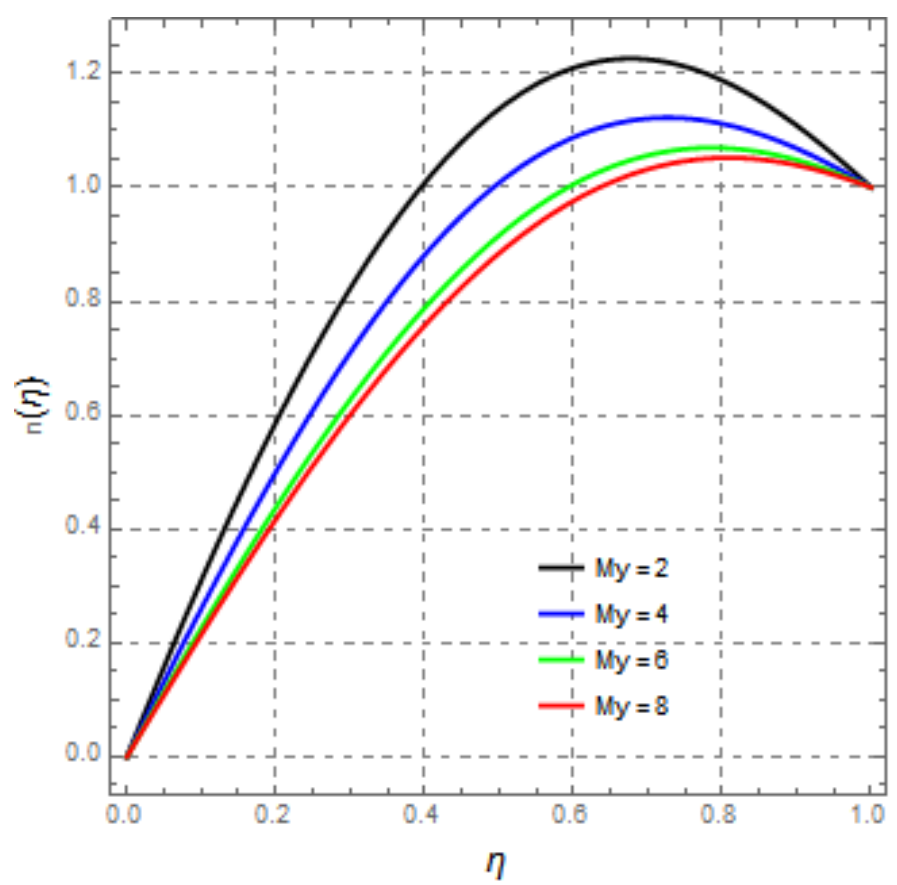

Figure 18. Observing the effect of magnetic strength parameter $M_{y}$ on $n(\eta)$ with $S_{z}=-0.5, M_{z}=$ $-1.5, R_{m}=-1, S=1, R_{d}=-1, D_{u}=2, P_{r n}=-0.1, S_{r t}=-0.2$ and $S_{c h}=-1$. 

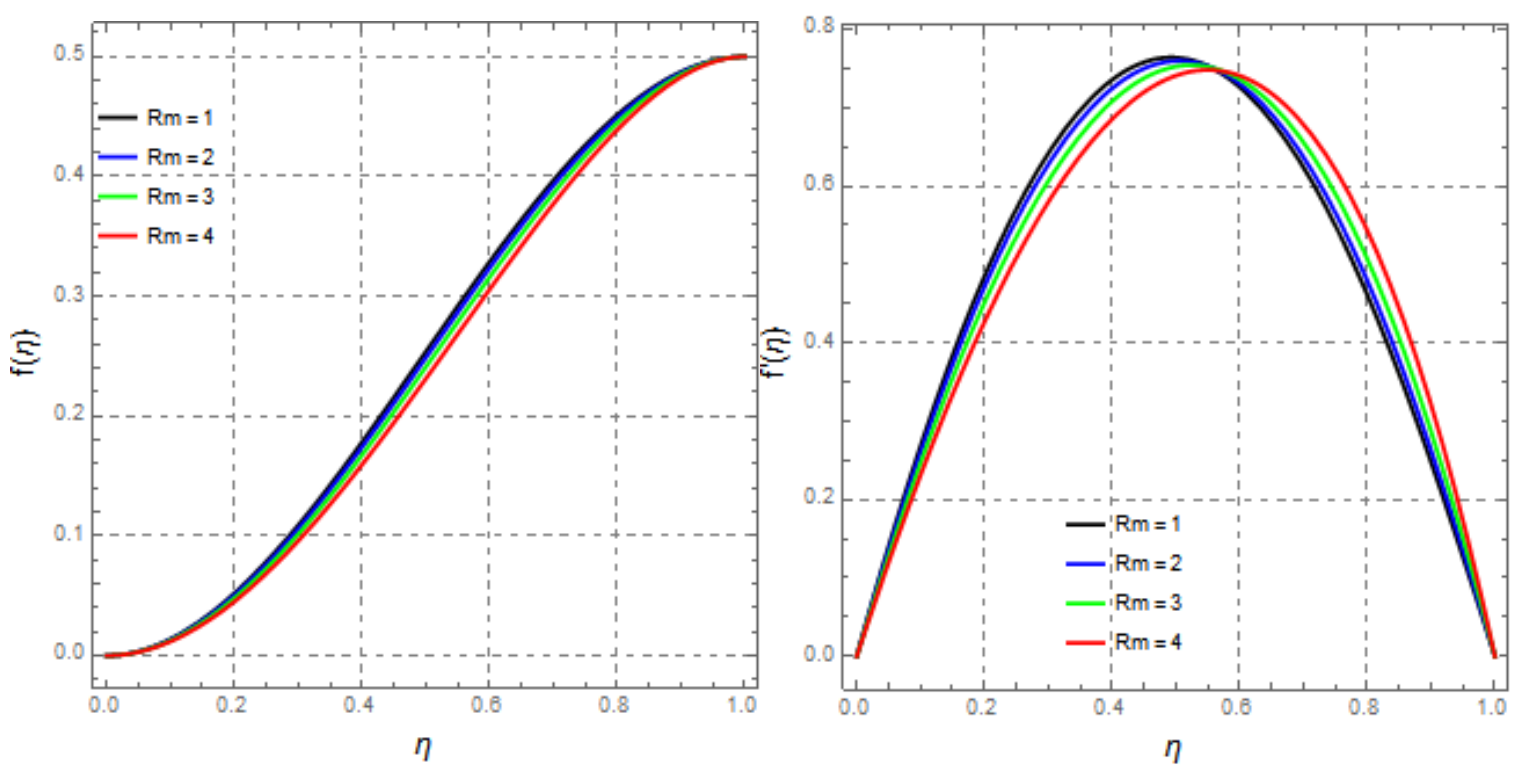

Figure 19. Observing the effect of magnetic Reynold number $R_{m}$ on $f(\eta)$ and $f^{\prime}(\eta)$ with $S_{z}=-1.5, M_{z}=-1.5, M_{y}=3$, $S=1, R_{d}=-1, D_{u}=5, P_{r n}=1, S_{r t}=3$ and $S_{c h}=1$.
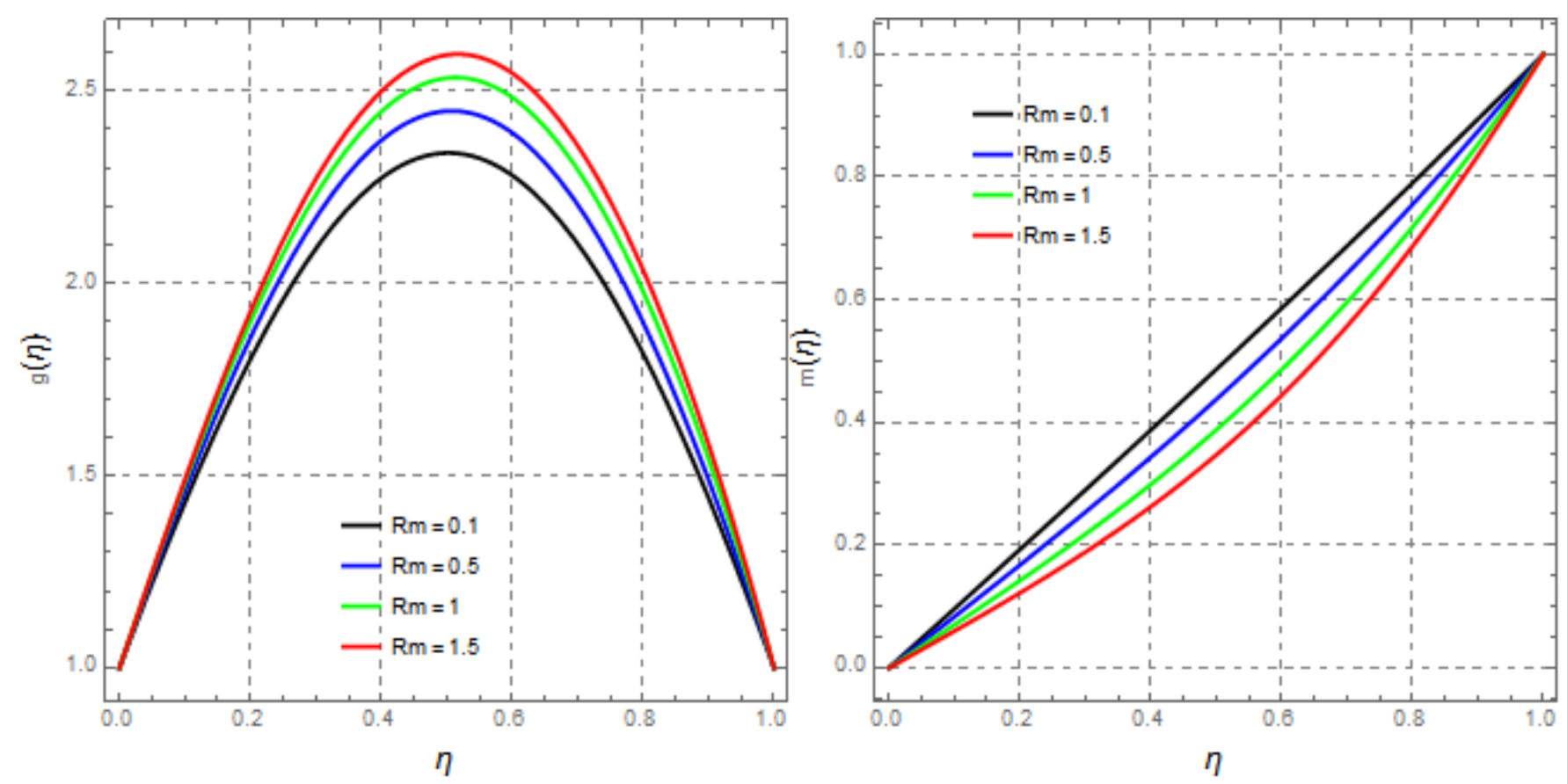

Figure 20. Observing the effect of magnetic Reynold number $R_{m}$ on $g(\eta)$ and $m(\eta)$ with $S_{z}=-1.5, M_{z}=-0.75, M_{y}=1$, $S=1, R_{d}=-1, D_{u}=0.1, P_{r n}=-1, S_{r t}=-2$ and $S_{c h}=-0.5$.

For the x-component of velocity initially, a decrease in the velocity is located but starts increasing as $\eta \rightarrow 1$ maximum value of $f^{\prime}(\eta)$ is observed at the center. In the case of magnetic field component $m(\eta)$, with $R_{m}=0.1$, an almost linear profile is observed, whereas when increasing the value of $R_{m}$, the profile becomes parabolic. It is also shown that in increasing $R_{m}$, the profile for $m(\eta)$ decreases. However, a direct relationship is observed in the case of magnetic field component $n(\eta)$, i.e., when increasing the values of $R_{m}$, the profile of $n(\eta)$ also increases. Similarly, for larger values of $R_{m}$, the profile becomes more parabolic. Moreover, observing the effect of $R_{m}$ on $\theta(\eta)$ and $\phi(\eta)$, it can be seen that increasing the magnetic Reynold number has a direct effect on temperature distribution $\theta(\eta)$, while an inverse relation is observed in the case of concentration $\phi(\eta)$. 


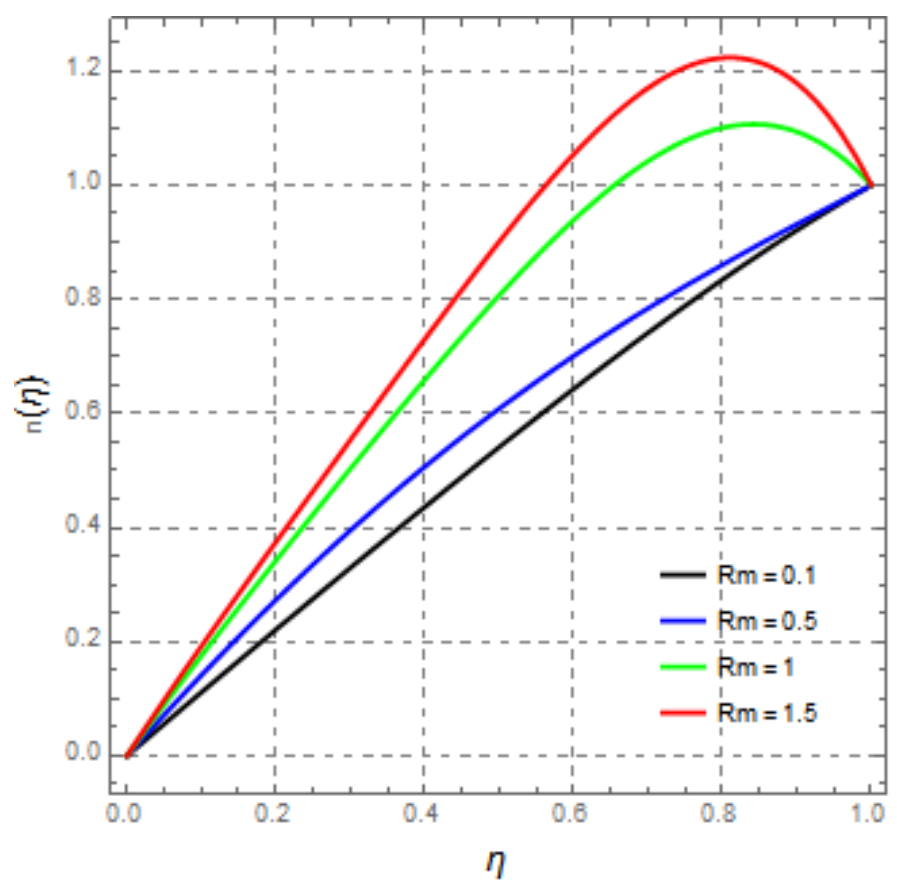

Figure 21. Observing the effect of magnetic Reynold number $R_{m}$ on $n(\eta)$ with $S_{z}=-1.5, M_{z}=0.5$, $M_{y}=1, S=1, R_{d}=1, D_{u}=0.1, P_{r n}=1, S_{r t}=2$ and $S_{c h}=0.5$.
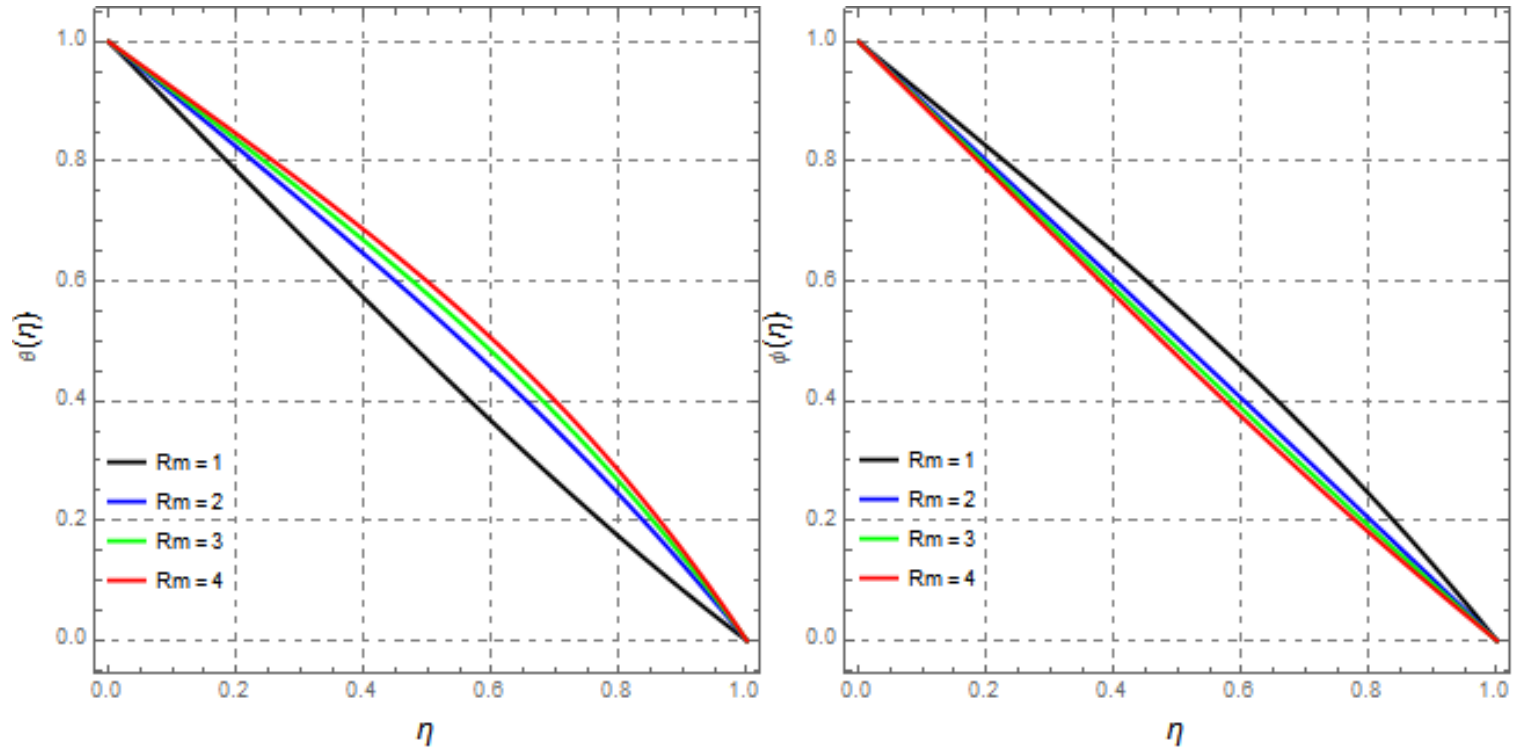

Figure 22. Observing the effect of magnetic Reynold number $R_{m}$ on $\theta(\eta)$ and $\phi(\eta)$ with $S_{z}=-1.5, M_{z}=-1.5, M_{y}=3$, $S=1, R_{d}=-1, D_{u}=5, P_{r n}=1, S_{r t}=3$ and $S_{c h}=1$.

Figure 23 illustrates the effect of the Dufour number on concentration and temperature distributions, showing that in both, cases $D_{u}$ has a direct effect on $\theta(\eta)$ and $\phi(\eta)$. Similarly, from Figure 24, it can be observed that the Prandtl number $P_{r n}$ has a direct relation with $\theta(\eta)$, as increasing $P_{r n}$ increases the temperature $\theta(\eta)$, whereas an inverse relation is observed in the case of $\phi(\eta)$, i.e., increasing $P_{r n}, \phi(\eta)$ shows a decreasing effect. Figure 25 depicts the Soret effect on temperature and concentration: it can be seen that in increasing the Soret number, heat transfer increases but mass transfer decreases. On the other hand, the opposite case is seen in the case of radiation parameter $R_{d}$ in Figure 26, i.e., increasing $R_{d}$, $\theta(\eta)$ increases while $\phi(\eta)$ decreases. Further, for the Schmidt number, an inverse relation is observed for $\theta(\eta)$, whereas a direct relation can be seen for $\phi(\eta)$, as seen from Figure 27.
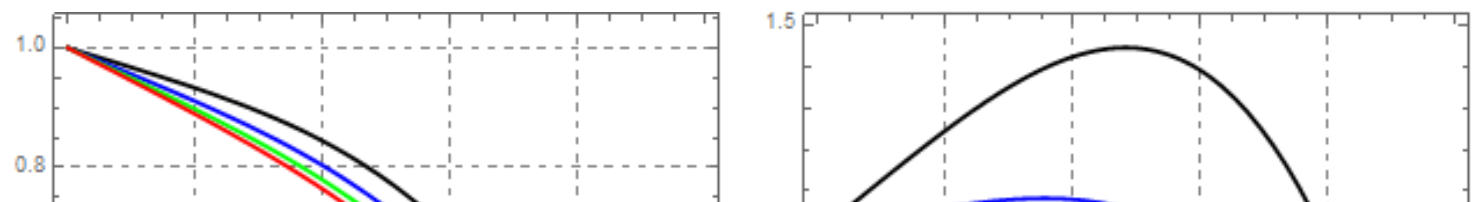

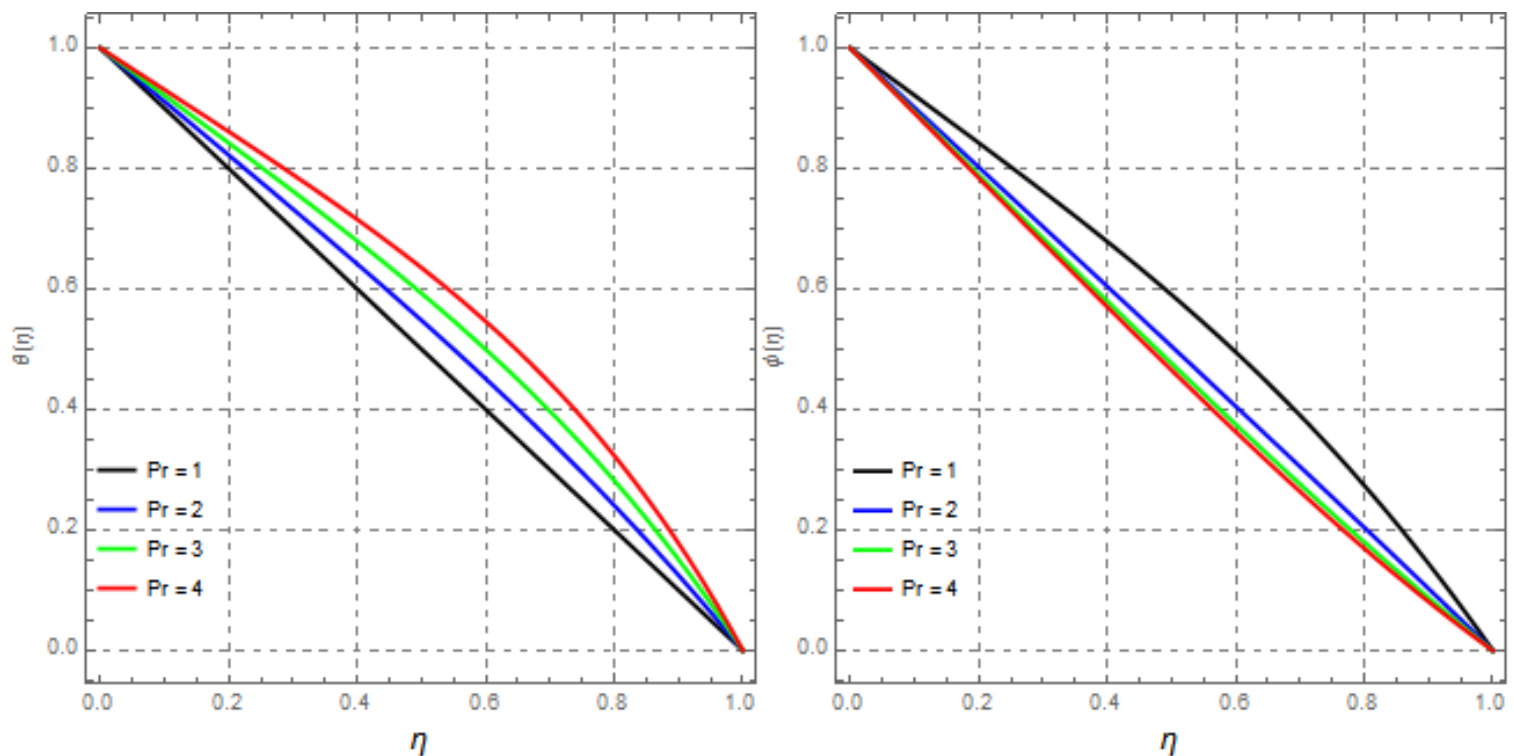

Figure 24. Observing the effect of Prandtl number $P_{r n}$ on $\theta(\eta)$ and $\phi(\eta)$ with $S_{z}=1.5, M_{z}=1.5, M_{y}=3, R_{m}=1, S=1$, $R_{d}=2, D_{u}=1, S_{r t}=2$ and $S_{c h}=1$.
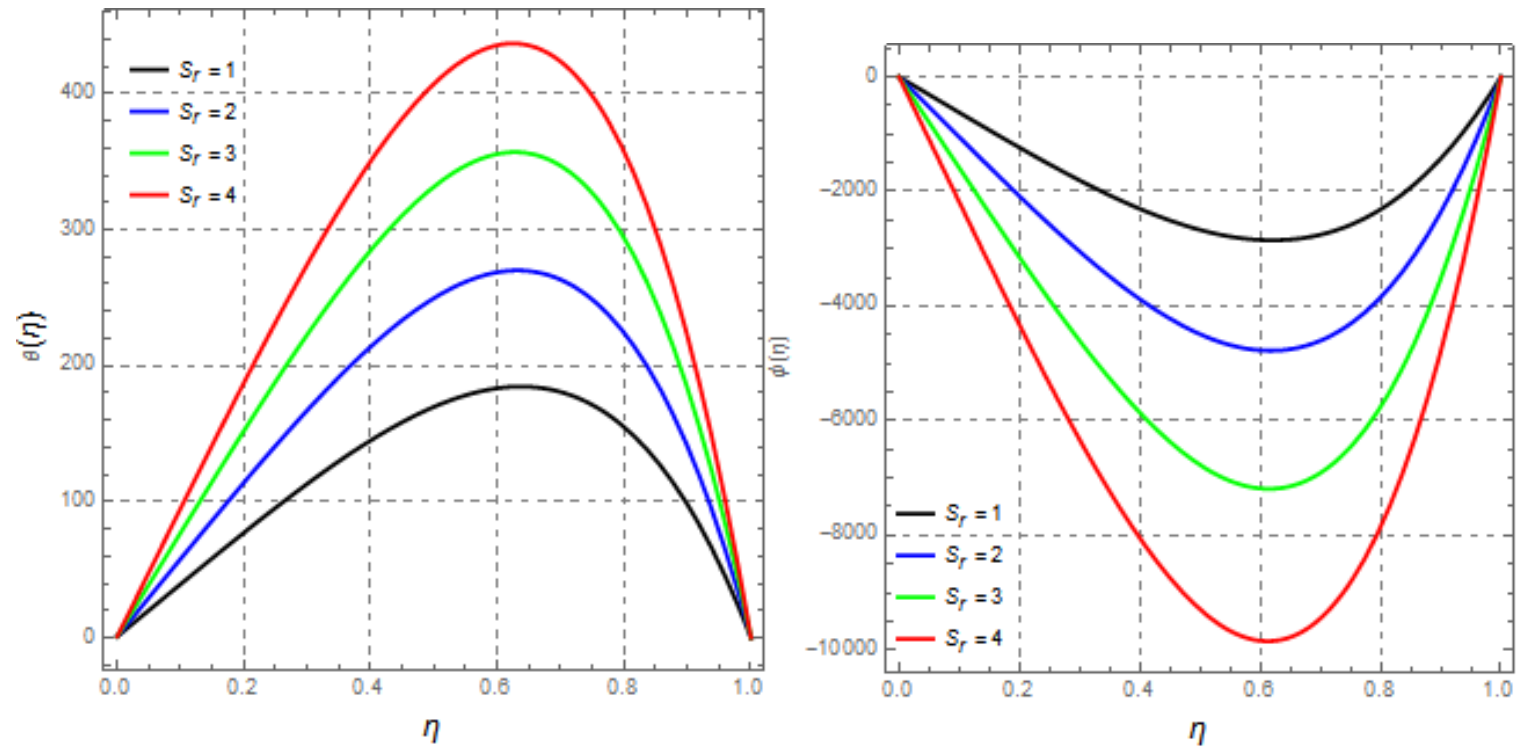

Figure 25. Observing the effect of Soret number $S_{r t}$ on $\theta(\eta)$ and $\phi(\eta)$ with $S_{z}=-1.5, M_{z}=-1.5, M_{y}=3, R_{m}=0.5, S=1$, $R_{d}=-1, D_{u}=0.1, P_{r n}=5$ and $S_{c h}=1$. 

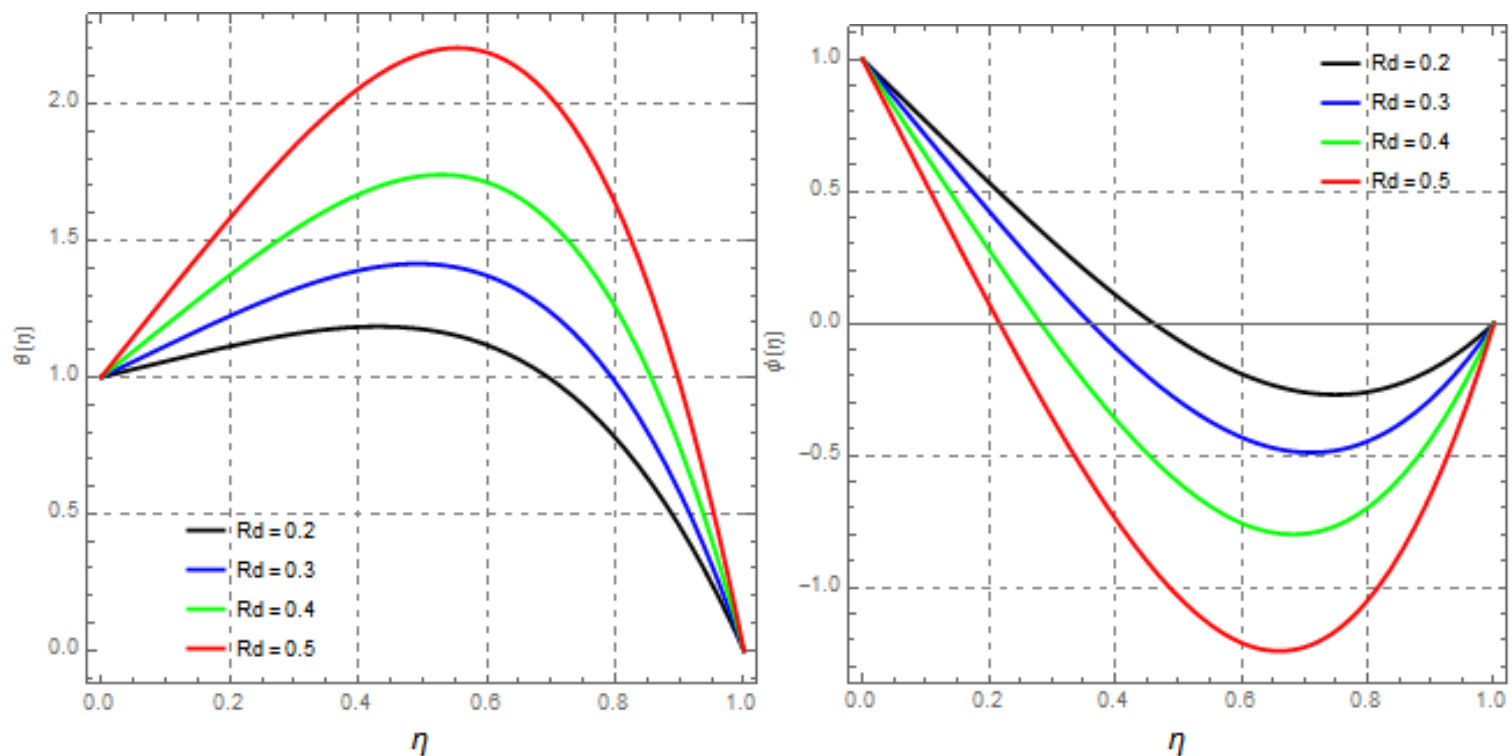

Figure 26. Observing the effect of radiation parameter $R_{d}$ on $\theta(\eta)$ and $\phi(\eta)$ with $S_{z}=-1.5, M_{z}=-1.5, M_{y}=3, R_{m}=-0.5$, $S=1, D_{u}=2, P_{r n}=1.5, S_{r t}=2$ and $S_{c h}=-1$.
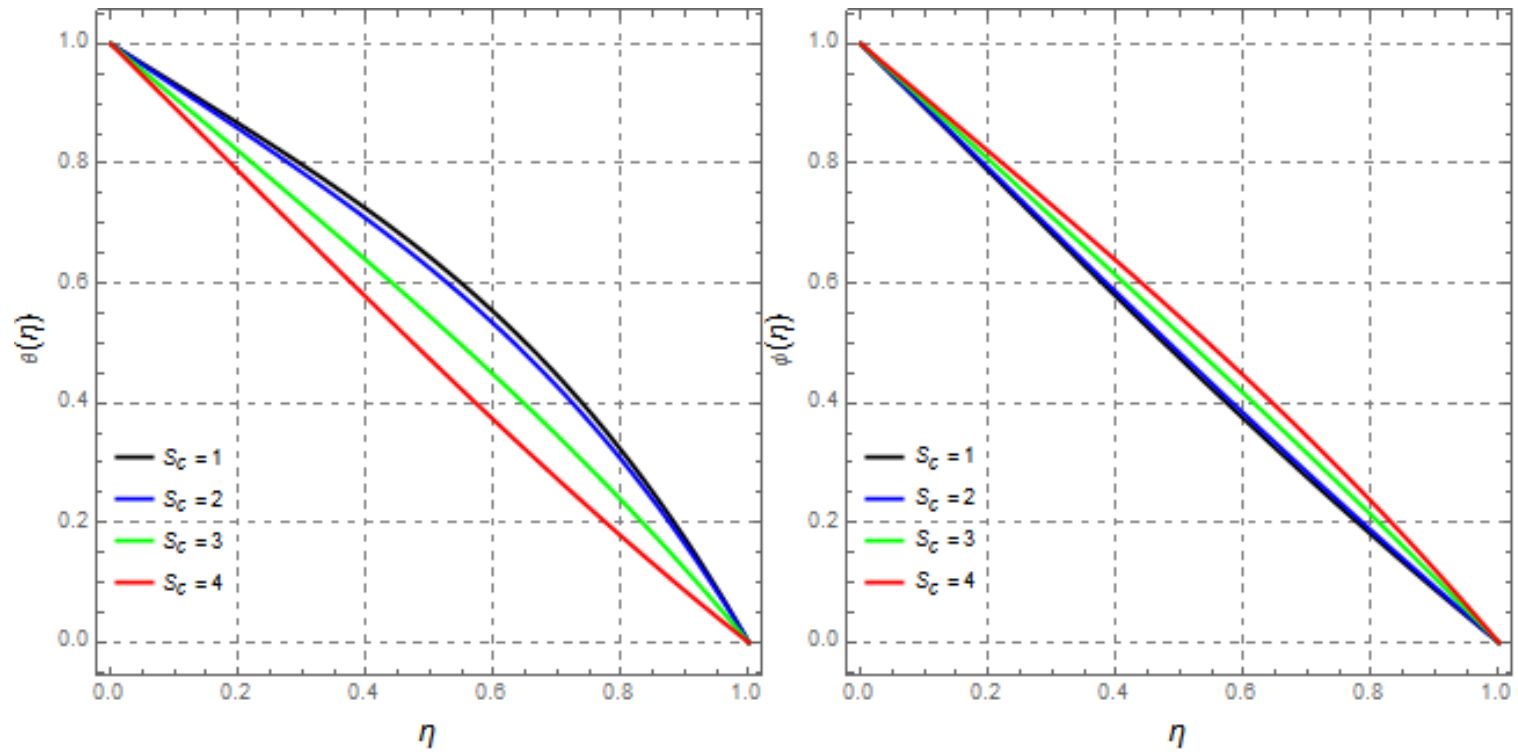

Figure 27. Observing the effect of Schmidt number $S_{c h}$ on $\theta(\eta)$ and $\phi(\eta)$ with $S_{z}=-1.5, M_{z}=-1.5, M_{y}=3, R_{m}=-0.5$, $S=1, R_{d}=-0.5, D_{u}=5, P_{r n}=1$, and $S_{r t}=2$.

\section{Conclusions}

Heat and mass transfer for 3D squeezing MHD flow of viscous fluids is studied in this chapter; flow is considered between two plates rotating at the same angular velocities. The influence of a varying magnetic field is introduced, and the phenomenon is represented using linked governing equations, such as continuity, Navier-Stokes, magnetic field energy, and transport equations. They are then translated to ordinary differential equations using the similarity transformation and solved using the analytical technique (HAM) in Mathematica package BVPh 2.0. The influence of varied parameters on velocity, magnetic field, concentration and temperature distribution was observed by graphs and tables. Below are some conclusions made from the above analysis: 
- It is determined that increasing the squeeze effect on the upper plate generates an increase in flow velocity along the $y$ - and $z$-axis, while the velocity along the $x$-axis initially increases but then decreases in the upper domain as $(\eta \rightarrow 1)$.

- It was also found that increasing the squeeze Reynold number of the magnetic field component reduced the magnetic field's effect along the z-component while increasing the effect along the y-component.

- While squeeze number has a direct effect on both concentration and temperature distribution, an increase in squeeze Reynold number causes an increase in both temperature and mass transfer.

- Moreover, raising the magnetic field strength parameter $M_{z}$, which is the strength of the magnetic field along the z-axis, results in a drop in fluid velocity along the z-axis, yet velocity along the y-axis shows a gradual increase by increasing $M_{z}$. However, along the $\mathrm{x}$-axis, firstly a decrease in the velocity component is observed, but as $\eta \rightarrow 1$, the velocity begins to increase.

- The magnetic field strength parameter $M_{z}$ has a direct relationship with the magnetic field component along the $z$-axis, i.e., increasing the value of $M_{z}$ has a increasing effect on the value of magnetic field along the z-component. A direct relationship can also be detected along the y-axis.

- $\quad$ Further, for a temperature distribution, increasing $M_{z}$ shows that $\theta(\eta)$ increases, but $\phi$ decreases by increasing $M_{z}$.

- Further, it is concluded that raising the y-component of the magnetic field strength parameter causes a decrease in the velocity of the fluid along the y-axis and the effect of the magnetic field along the y-axis.

- It is concluded that increasing the magnetic Reynold number $R_{m}$ causes a decrease in flow velocity along the z-axis. On the other hand, raising $R_{m}$ has an increasing effect on velocity along $\mathrm{y}$. The flow velocity along the $\mathrm{x}$-axis initially shows a diminishing pattern, but when $\eta \rightarrow 1$ increases, the effect increases.

- It is also observed that for the magnetic field, increasing the magnetic Reynold number shows a decrease in the value of the magnetic field along the z-axis, whereas an increasing effect is observed along the y-axis.

- Furthermore, it is also concluded that increasing the magnetic Reynold number shows an increase in the heat transfer, where a decrease in the mass transfer is observed.

- The effect of the Dufour number on concentration and temperature distribution is also observed, and it is concluded that in both cases, $D_{u}$ has a direct effect on mass and temperature distribution, as increasing the Dufour number increases both mass and heat transfer.

- It is also concluded that an increase in the value of the Prandtl number causes an increase in the value of $\theta(\eta)$, but a decrease in the value of $\phi(\eta)$ is observed.

- For the Soret number, it can be seen that in increasing the Soret number, heat transfer increases but mass transfer decreases. Thus, it has a direct effect on $\theta(\eta)$ and an inverse effect on $\phi(\eta)$.

- It is further concluded that opposite behavior is observed in case of the radiation parameter's effect on temperature distribution, i.e., in increasing its value, $\theta(\eta)$ decreases, while in case of mass transfer, $\phi(\eta)$ increases with an increase in the value of the radiation parameter.

- For the Schmidt number, it is concluded that with an increasing Schmidt number, both heat and mass transfer values increase.

Author Contributions: Conceptualization, M.K.A.; methodology, A.K.; formal analysis, K.B.; funding acquisition, S.N. All authors have read and agreed to the published version of the manuscript.

Funding: This research received no external funding

Institutional Review Board Statement: Not applicable.

Informed Consent Statement: Not applicable. 


\section{Data Availability Statement: Not applicable.}

Conflicts of Interest: The authors declare no conflict of interest.

\section{Nomenclature}

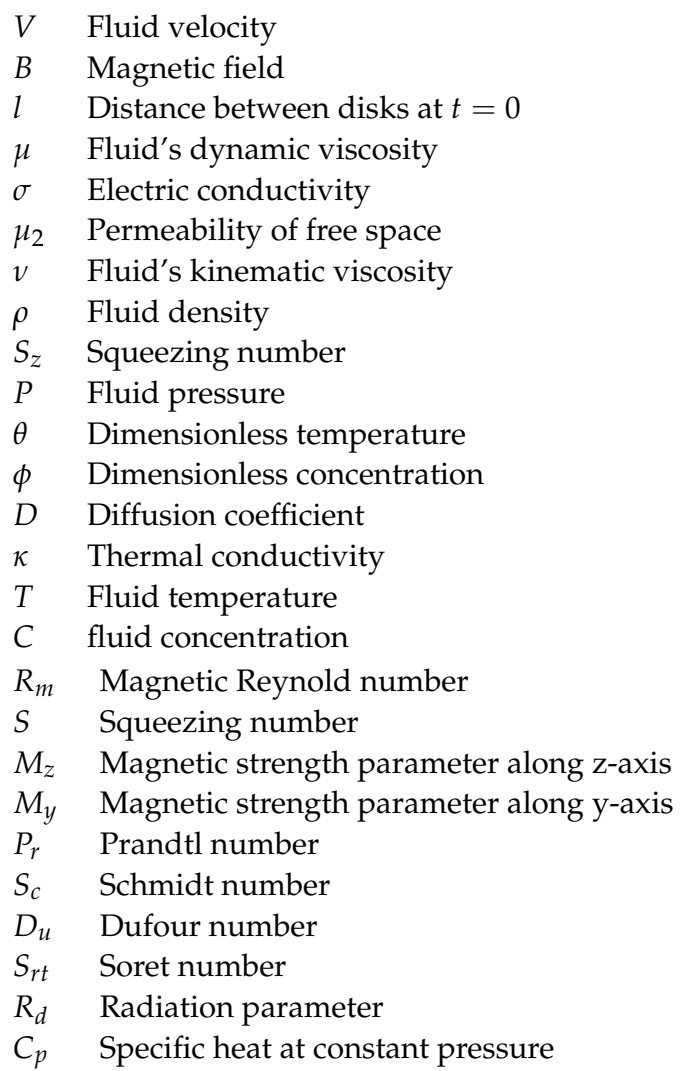

\section{References}

1. Siddiqui, A.M.; Irum, S.; Ansari, A.R. Unsteady squeezing flow of a viscous MHD fluid between parallel plates, a solution using the homotopy perturbation method. Math. Model. Anal. 2008, 13, 565-576. [CrossRef]

2. Sweet, E.; Vajravelu, K.; Van Gorder, R.A.; Pop, I. Analytical solution for the unsteady MHD flow of a viscous fluid between moving parallel plates. Commun. Nonlinear Sci. Numer. Simul. 2011, 16, 266-273. [CrossRef]

3. Ramachandra Murty, P.S.; Prakash, G.B. MHD Two-Fluid Flow and Heat Transfer between Two Inclined Parallel Plates in a Rotating System. Hindawi Publ. Corp. 2014, 2014, 256898. [CrossRef] [PubMed]

4. Onyango, E.R.; Kinyanjui, M.; Kimathi, M. Unsteady hydromagnetic flow between parallel plates both moving in the presence of a constant pressure gradient. Int. J. Eng. Sci. Innov. Technol. (IJESIT) 2017, 6. Available online: http://ir.mksu.ac.ke/handle/1234 $56780 / 2181$ (accessed on 14 August 2021).

5. Khan, M.S.; Shah, R.A.; Khan, A. Effect of variable magnetic field on the flow between two squeezing plates. Eur. Phys. J. Plus 2019, 134, 219. [CrossRef]

6. Muhammad, T.; Hayat, T.; Alsaedi, A.; Qayyum, A. Hydromagnetic unsteady squeezing flow of Jeffrey fluid between two parallel plates. Chin. J. Phys. 2017, 55, 1511-1522. [CrossRef]

7. Verma, P.D.; Mathur, A. Magenetohydrodynamic Flow between Two Parallel Plates, One in Uniform Motion and the Other at Rest with Uniform Suction at the Stationary Plate. Proc. Indian Natl. Sci. Acad. USA 1969, 35, 4.

8. Hayat, T.; Sajjad, R.; Alsaedi, A.; Muhammad, T.; Ellahi, R. On squeezed flow of couple stress nanofluid between two parallel plates. Results Phys. 2017, 7, 553-561. [CrossRef]

9. Linga Raju, T. MHD heat transfer two-ionized fluids flow between parallel plates with Hall currents. Results Eng. 2019, 4, 100043. [CrossRef]

10. Hamza, E.A. The magnetohydrodynamic effects on a fluid film squeezed between two rotating surfaces. J. Phys. Appl. Phys. 1991, 24, 547-554. [CrossRef]

11. Das, S.; Maji, S.L.; Guria, M.; Jana, R.N. Unsteady MHD Couette flow in a rotating system. Math. Comput. Model. 2009, 50, 1211-1217. [CrossRef]

12. Parter, S.V.; Rajagopal, K.R. Swirling Flow between Rotating Plates. In The Breadth and Depth of Continuum Mechanics; Springer: Berlin/Heidelberg, Germany, 1986; ISBN 978-3-642-61634-1. 
13. Rajagopal, K.R.; Parter, S.V. Remarks on the Flow between Two Parallel Rotating Plates; Technical Summary Report Wisconsin University, Madison; Mathematics Research Center: Madison,WI, USA, 1984.

14. Rajagopal, K.R. The flow of a second order fluid between rotating parallel plates. J. Non-Newton. Fluid Mech. 1981, 9, 185-190. [CrossRef]

15. Rajat, T.; Gauri, S.; Manoj, M. Double diffusive flow of a hydromagnetic nanofluid in a rotating channel with Hall effect and viscous dissipation: Active and passive control of nanoparticles. Adv. Powder Technol. 2017, $28,10$.

16. Hughes, W.; Elco, R. Magnetohydrodynamic lubrication flow between parallel rotating disks. J. Fluid Mech. 1962, 13, 21-32. [CrossRef]

17. Elshekh, S.S.; Elhady, M.K.A.; Ibrahim, F.N. Fluid film squeezed between two rotating disks in the presence of a magnetic field. Int. J. Eng. Sci. 1996, 34, 1183-1195. [CrossRef]

18. Shah, R.A.; Khan, A.; Shuaib, M. On the study of flow between unsteady squeezing rotating discs with cross diffusion effects under the influence of variable magnetic field. Heliyon 2018, 4, e00925. [CrossRef] [PubMed]

19. Ganji, D.D.; Abbasi, M.; Rahimi, J.; Gholami, M.; Rahimipetroudi, I. On the MHD squeeze flow between two parallel disks with suction or injection via HAM and HPM. Front. Mech. Eng. 2014, 9, 270-280. [CrossRef]

20. Khan, A.; Shah, R.A.; Shuaib, M.; Ali, A. Fluid dynamics of the magnetic field dependent thermosolutal convection and viscosity between coaxial contracting discs. Results Phys. 2018, 9, 923-938. [CrossRef]

21. Munawar, S.; Mehmood, A.; Ali, A. Three-dimensional squeezing flow in a rotating channel of lower stretching porous wall. Comput. Math. Appl. 2012, 64, 1575-1586. [CrossRef]

22. Alzahrani Abdullah, K.; Zaka, U.M.; Taseer, M. Numerical Treatment for 3D Squeezed Flow in a Rotating Channel With Soret and Dufour Effects. Front. Phys. 2020, 8, 201. [CrossRef]

23. Shah, Z.; Gul, T.; Islam, S.; Khan, M.A.; Bonyah, E.; Hussain, F.; Mukhtar, S.; Ullah, M. Three dimensional third grade nanofluid flow in a rotating system between parallel plates with Brownian motion and thermophoresis effects. Results Phys. 2018, 10, 36-45. [CrossRef]

24. Riasat, S.; Ramzan, M.; Kadry, S.; Chu, Y.-M. Significance of Magnetic Reynolds number in a three-dimensional squeezing Darcy-Forchheimer hydromagnetic nanofluid thin-film flow between two rotating disks. Sci. Rep. 2020, 10, 17208. [CrossRef]

25. Fiza, M.; Alsubie, A.; Ullah, H.; Hamadneh, N.N.; Islam, S.; Khan, I. Three-Dimensional Rotating Flow of MHD Jeffrey Fluid Flow between Two Parallel Plates with Impact of Hall Current. Math. Probl. Eng. 2021, 2021, 6626411. [CrossRef]

26. Srinivas, S.; Reddy, A.S.; Ramamohan, T.R.; Shukla, A.K. Influence of heat transfer on MHD flow in a pipe with expanding or contracting permeable wall. Ain Shams Eng. J. 2014, 5, 817-830. [CrossRef]

27. Sparrow, E.M.; Cess, R.D. The effect of a magnetic field on free convection heat transfer. Int. J. Heat Mass Transf. 1961, 3, 267-274. [CrossRef]

28. Khaled, A.R.A.; Vafai, K.Hydromagnetic squeezed flow and heat transfer over a sensor surface. Int. J. Eng. Sci. 2004, 42, 509-519. [CrossRef]

29. Tasawar, H.; Muhammad, Q.; Zaheer, A. Radiation and Mass Transfer Effects on the Magnetohydrodynamic Unsteady Flow Induced by a Stretching Sheet. Zeitschrift für Naturforschung A 2010, 65, 231-239.

30. Mahmood, M.; Asghar, S.; Hossain, M.A. Squeezed flow and heat transfer over a porous surface for viscous fluid. Heat Mass Transfer 2007, 44, 165-173. [CrossRef]

31. Tsai, R.; Huang, J.S. Heat and mass transfer for Soret and Dufour's effects on Hiemenz flow through porous medium onto a stretching surface. Int. J. Heat Mass Transf. 2009, 52, 2399-2406. [CrossRef]

32. Mustafa, M.; Hayat, T.; Obaidat, S. On heat and mass transfer in the unsteady squeezing flow between parallel plates. Meccanica 2012, 47, 1581-1589. [CrossRef]

33. Yasmin, A.; Ali, K.; Ashraf, M. Study of Heat and Mass Transfer in MHD Flow of Micropolar Fluid over a Curved Stretching Sheet. Sci. Rep. 2020, 10, 4581. [CrossRef] [PubMed]

34. Sheikholeslami, M.; Ganji, D.D. Three dimensional heat and mass transfer in a rotating system using nanofluid. Powder Technol. 2014, 253, 789-796. [CrossRef]

35. Bilal, S.; Rehman, M.; Noeiaghdam, S.; Ahmad, H.; Akgul, A. Numerical Analysis of Natural Convection Driven Flow of a Non-Newtonian Power-Law Fluid in a Trapezoidal Enclosure with a U-Shaped Constructal. Energies 2021, 14, 5355. [CrossRef]

36. Noeiaghdam, S.; Sidorov, D. Integral equations: Theories, Approximations and Applications. Symmetry 2021, 13, 1402. [CrossRef]

37. Ghomanjani, F.; Noeiaghdam, S. Application of Said Ball curve for solving fractional differential-algebraic equations. Mathematics 2021, 9, 1926. [CrossRef]

38. Zarei, E.; Noeiaghdam, S. Advantages of the Discrete Stochastic Arithmetic to Validate the Results of the Taylor Expansion Method to Solve the Generalized Abel's Integral Equation. Symmetry 2021, 13, 1370. [CrossRef]

39. Izadi, M.; Yuzbasi, S.; Noeiaghdam, S. Approximating solutions of non-linear Troesch's problem via an efficient quasi-linearization Bessel approach. Mathematics 2021, 9, 1841. [CrossRef]

40. Zhao, Y.; Liao, S. User Guide to BVPh 2.0. 2002. Available online: http:// numericaltank.sjtu.edu.cn/BVPh.htm (accessed on 14 August 2021). 